\title{
Implementation of supersymmetric processes in the HERWIG event generator*
}

\author{
Stefano Moretti \\ Theory Division, CERN and IPPP, University of Durham, UK \\ E-mail: Stefano.Moretti@cern.ch

\section{Kosuke Odagiri} \\ Theory Group, KEK, Japan \\ E-mail: odagirik@post.kek.jp

\section{Peter Richardson} \\ Cavendish Laboratory and DAMTP, University of Cambridge, UK \\ E-mail: richardn@hep.phy.cam.ac.uk
}

\section{Michael H. Seymour}

Department of Physics \& Astronomy, University of Manchester, UK E-mail: M.Seymour@rl.ac.ut

\section{Bryan R. Webber}

Cavendish Laboratory, University of Cambridge, UK

E-mail: webber@hep.phy.cam.ac.ul

ABSTRACT: We describe the implementation of supersymmetric processes in the HERWIG Monte Carlo event generator. We define relevant parameter and mixing conventions and list the hard scattering matrix elements. Our implementation is based on the Minimum Supersymmetric Standard Model, with the option of R-parity violation. The sparticle spectrum is completely general. Both hadron-hadron and lepton-lepton collisions are covered. This article supplements a separate publication in which the general features of HERWIG 6.2 are described, and updates the treatment of supersymmetry to version 6.4.

KEYwords: Supersymmetry Phenomenology, Supersymmetric Standard Model, Higgs Physics.

${ }^{*}$ Work supported in part by the U.K. Particle Physics and Astronomy Research Council and by the EU Fourth Framework Programme "Training and Mobility of Researchers", Network "Quantum Chromodynamics and the Deep Structure of Elementary Particles", contract FMRX-CT98-0194 (DG 12 - MIHT). 


\section{Contents}

1. Introduction 1

2. Parameter and mixing conventions 2

2.1 Particle content 3

2.2 Parameter conventions

2.3 Mixing conventions

2.3.1 Charginos

2.3 .2 Neutralinos 8

2.3.3 Left-right sfermion mixing 10

2.3.4 Higgs bosons 11

3. Matrix elements 12

3.1 Notation 12

3.2 Sparton pair production 13

3.3 Slepton pair production 13

3.4 Gaugino production 14

3.5 Neutral Higgs production 16

3.6 Charged Higgs production 21

3.7 R-parity violating processes 23

3.7.1 Single gaugino production 24

3.7.2 Slepton production in association with a gauge boson 25

3.7.3 Slepton production in association with a Higgs boson 25

3.7.4 SM particle production by sparticle exchange 26

3.8 Decay matrix elements 27

4. Conclusions 27

A. Process codes 28

\section{Introduction}

HERWIG $^{1}$ [1] is a general-purpose event generator for high-energy processes. A major new feature is the inclusion of supersymmetric (SUSY) processes, made available for the first time in HERWIG 6.1 and extended in subsequent versions. This article describes the implementation, defines the parameter and mixing conventions, and lists the hard scattering matrix elements. For the details of the input file format and for a more general description of our implementation, as well as the description of the Standard Model (SM)

\footnotetext{
${ }^{1}$ The latest version of the program, together with all relevant information, is always available via the official HERWIG page: http://hepwww.rl.ac.uk/theory/seymour/herwig/.
} 
physics implemented in the generator, the reader should consult ref. [1]. This article supplements that paper and extends it to version 6.4. The exact version number of the code described in this article is 6.400 .

The key features of the HERWIG 6.4 supersymmetry implementation are as follows:

- A general Minimal Supersymmetric Standard Model (MSSM) particle spectrum.

- Both hadron-hadron and lepton-lepton collisions.

- MSSM $2 \rightarrow 2$ sparticle production subprocesses.

- $2 \rightarrow 1,2 \rightarrow 2$ and $2 \rightarrow 3$ Two Higgs Doublet Model (2HDM) Higgs boson production subprocesses in hadron-hadron collisions, and $2 \rightarrow 2$ Higgs boson production processes in lepton-lepton collisions.

- All R-parity violating $2 \rightarrow 2$ scattering subprocesses in hadron-hadron collisions that can, but do not necessarily, have an $s$-channel resonance, and $2 \rightarrow 2$ scattering processes in lepton-lepton collisions, not necessarily with an $s$-channel resonance.

- Cascade decay of heavy objects according to branching fractions taken from an input data file, incorporating 3-body and 4-body matrix elements.

- Colour coherence between emitted partons.

- Spin correlations in $2 \rightarrow 2 \mathrm{R}$-parity conserving production processes and all cascade decay processes.

- Beam polarisation in lepton-lepton collisions.

The masses, total decay widths and branching fractions, the mixings of gauginos, third generation sfermions and Higgs bosons, the bilinear, trilinear and R-parity violating trilinear couplings, are read in from a data file. A separate program (ISAWIG) is available (from the HERWIG web page) to convert the data from ISAJET [2] and HDECAY [3] to a form suitable for HERWIG and modify it to include the R-parity violating sector.

In our implementation, supersymmetric particles do not radiate gluons. This assumption is reasonable if the decay lifetimes of the coloured sparticles (spartons) are much shorter than the QCD confinement scale. In particular, a stable gluino Lightest Supersymmetric Particle (LSP) is not simulated. The simulation of a long-lived light top squark would neglect effects due to gluon emission and hadronization.

CP-violating phases are not included for either the mixings or the couplings.

$\mathrm{R}$-parity violating lepton-gaugino and slepton-Higgs mixing is not considered.

\section{Parameter and mixing conventions}

There are several different conventions that are commonly adopted in the literature for the MSSM lagrangian. While most authors have chosen to follow the conventions used in refs. [ [, 5], other conventions, for example ref. [2, are also commonly used. The conventions 


\begin{tabular}{|c|c|c|}
\hline spin 0 & spin $1 / 2$ & spin 1 \\
\hline \hline $\begin{array}{c}\text { down-type squarks } \\
\left(\widetilde{d}_{L}, \widetilde{d}_{R}, \widetilde{s}_{L}, \widetilde{s}_{R}, \widetilde{b}_{1}, \widetilde{b}_{2}\right)\end{array}$ & $\begin{array}{c}\text { down-type quarks } \\
(d, s, b)\end{array}$ & \\
\hline up-type squarks & up-type quarks & \\
$\left(\widetilde{u}_{L}, \widetilde{u}_{R}, \widetilde{c}_{L}, \widetilde{c}_{R}, \widetilde{t}_{1}, \widetilde{t}_{2}\right)$ & $(u, c, t)$ & \\
\hline charged sleptons & charged leptons & \\
$\left(\widetilde{e}_{L}, \widetilde{e}_{R}, \widetilde{\mu}_{L}, \widetilde{\mu}_{R}, \widetilde{\tau}_{1}, \widetilde{\tau}_{2}\right)$ & $\left(e^{-}, \mu^{-}, \tau^{-}\right)$ & \\
\hline sneutrinos & neutrinos & \\
$\left(\widetilde{\nu}_{e}, \widetilde{\nu}_{\mu}, \widetilde{\nu}_{\tau}\right)$ & $\left(\nu_{e}, \nu_{\mu}, \nu_{\tau}\right)$ & \\
\hline & gluino & gluon \\
\hline neutral Higgs & neutralinos & photon and $Z^{0}$ \\
$\left(h^{0}, H^{0}, A^{0}\right)$ & $\widetilde{\chi}_{i}^{0}(i=1-4)$ & $\left(\gamma, Z^{0}\right)$ \\
\hline charged Higgs & charginos & $W^{ \pm}$ \\
$H^{+}$ & $\widetilde{\chi}_{i}^{+}(i=1-2)$ & $W^{+}$ \\
\hline \multicolumn{2}{|c|}{ Goldstino } & \\
\hline
\end{tabular}

Table 1: Particle content of the MSSM, expressed in terms of its mass eigenstates. The Goldstino $\widetilde{G}$ is the spin- $1 / 2$ component of the gravitino. We neglect mixing for the first two generation sfermions.

we have used in general follow those of refs. 近 5]. In this section we present our conventions for the mixing matrices for the charginos, neutralinos and sfermions, together with the conversion between the conventions of refs. [2, 田 5 .

\subsection{Particle content}

The MSSM particle content, expressed in terms of its mass eigenstates, is given in table \&.

In defining our conventions we need to write the particle content in terms of the interaction eigenstates. This is shown in table 2. Superscripts $c$ refer to charge conjugation, which, stated in the four-component notation, is defined by:

$$
\psi^{c}=C \bar{\psi}^{T}=i \gamma^{2} \gamma^{0} \bar{\psi}^{T}
$$

We adopt the chirality basis. The two-component case, which we refer to when discussing mixing conventions, follows trivially. Acting on chirality-left and chirality-right two-component spinors, we have:

$$
\begin{aligned}
& \left(\phi_{L}\right)^{c}=i \sigma^{2} \phi_{L}^{*}=\varepsilon_{i j} \phi_{j L}^{*}, \\
& \left(\phi_{R}\right)^{c}=-i \sigma^{2} \phi_{R}^{*}=-\varepsilon_{i j} \phi_{j R}^{*} .
\end{aligned}
$$

The Levi-Civita tensor $\varepsilon_{i j}$ is defined by $\varepsilon_{12}=-\varepsilon_{21}=1, \varepsilon_{11}=\varepsilon_{22}=0$. 


\begin{tabular}{|c|c|c|}
\hline spin 0 & $\operatorname{spin} 1 / 2$ & spin 1 \\
\hline $\begin{array}{c}\widetilde{Q}=\left(\begin{array}{c}\widetilde{U}_{L} \\
\widetilde{D}_{L}\end{array}\right) \\
\widetilde{D}=\widetilde{D}_{R}^{*} \\
\widetilde{U}=\widetilde{U}_{R}^{*}\end{array}$ & $\begin{array}{c}Q=\left(\begin{array}{l}u_{L} \\
d_{L}\end{array}\right) \\
D=d_{R}^{c} \\
U=u_{R}^{c}\end{array}$ & \\
\hline $\begin{array}{c}\widetilde{L}=\left(\begin{array}{c}\widetilde{\nu} \\
\widetilde{e}_{L}\end{array}\right) \\
\widetilde{E}=\widetilde{e}_{R}^{*}\end{array}$ & $\begin{array}{c}L=\left(\begin{array}{c}\nu \\
e_{L}\end{array}\right) \\
E=e_{R}^{c}\end{array}$ & \\
\hline & $\widetilde{g}$ & $g$ \\
\hline $\begin{array}{l}H_{1}=\left(\begin{array}{c}H_{1}^{0} \\
H_{1}^{-}\end{array}\right) \\
H_{2}=\left(\begin{array}{c}H_{2}^{+} \\
H_{2}^{0}\end{array}\right)\end{array}$ & $\begin{array}{c}\widetilde{B} \\
\widetilde{W}=\left(\begin{array}{l}\widetilde{W}^{+} \\
\widetilde{W}_{3}^{0} \\
\widetilde{W}^{-}\end{array}\right. \\
\widetilde{H}_{1}=\left(\begin{array}{c}\widetilde{H}_{1}^{0} \\
\widetilde{H}_{1}^{-}\end{array}\right. \\
\widetilde{H}_{2}=\left(\begin{array}{c}\widetilde{H}_{2}^{+} \\
\widetilde{H}_{2}^{0}\end{array}\right)\end{array}$ & $\begin{array}{c}B \\
W=\left(\begin{array}{c}W^{+} \\
W_{3}^{0} \\
W^{-}\end{array}\right)\end{array}$ \\
\hline & $\widetilde{G}$ & \\
\hline
\end{tabular}

Table 2: Field content of the MSSM, expressed in terms of its interaction eigenstates. Generation and colour indices are suppressed.

\subsection{Parameter conventions}

In terms of the scalar fields defined in table 2 , we write our scalar superpotential as follows:

$$
W_{s}=\varepsilon_{i j}\left[\mu H_{1}^{i} H_{2}^{j}+f_{\ell} H_{1}^{i} \widetilde{L}^{j} \widetilde{E}+f_{D} H_{1}^{i} \widetilde{Q}^{j} \widetilde{D}-f_{U} H_{2}^{i} \widetilde{Q}^{j} \widetilde{U}\right] .
$$

The above definition is consistent with refs. [5, 6]. The sign of the term proportional to the up-type quark coupling Yukawa matrix $f_{U}$ is such that the Yukawa couplings are positive.

For the R-parity violating scalar superpotential, we take:

$$
W_{\mathbb{R}_{\mathrm{p}}}=\varepsilon_{i j}\left[\kappa_{a} \widetilde{L}_{a}^{i} H_{2}^{j}+\frac{1}{2} \lambda_{a b c} \widetilde{L}_{a}^{i} \widetilde{L}_{b}^{j} \widetilde{E}_{c}+\lambda_{a b c}^{\prime} \widetilde{L}_{a}^{i} \widetilde{Q}_{b}^{j} \widetilde{D}_{c}\right]+\frac{1}{2} \lambda_{a b c}^{\prime \prime} \varepsilon_{c_{1} c_{2} c_{3}} \widetilde{U}_{a}^{c_{1}} \widetilde{D}_{b}^{c_{2}} \widetilde{D}_{c}^{c_{3}} .
$$

$a, b, c$ are the generation indices. In the last term, $c_{1}, c_{2}, c_{3}$ are the triplet colour indices and the totally antisymmetric tensor is defined by $\varepsilon_{123}=1$. Summation over colour indices is implicit in the other terms. We omit the bilinear term proportional to $\kappa_{a}$.

We introduce our soft SUSY-breaking bilinear and trilinear terms as follows:

$$
V_{I}=\varepsilon_{i j}\left[\mu B H_{1}^{i} H_{2}^{j}+f_{\ell} A_{\tau} H_{1}^{i} \widetilde{L}^{j} \widetilde{E}+f_{D} A_{b} H_{1}^{i} \widetilde{Q}^{j} \widetilde{D}-f_{U} A_{t} H_{2}^{i} \widetilde{Q}^{j} \widetilde{U}\right]+\text { (h.c.) . }
$$

This is consistent with the definition in ref. [0]. We neglect $A$ term contributions to the first two generations. The contribution of the potential to the lagrangian is $\mathcal{L}=-V$ in general. (h.c.) stands for hermitean conjugation. 
Our soft SUSY-breaking masses are defined schematically as follows:

$$
V_{M}=\sum_{\phi} M_{\phi}^{2} \phi^{\dagger} \phi+\sum_{\psi} M_{\psi} \bar{\psi} \psi
$$

where $\phi$ are the sfermion fields and $\psi$ are the gaugino and gluino fields in the fourcomponent notation. Summations over colour and weak isospin indices are implicit. We write the gaugino soft-breaking masses as $M_{\widetilde{B}}=M_{1}$ and $M_{\widetilde{W}}=M_{2}$. In the notation of refs. [4, 5], $M^{\prime}$ and $M$ are used instead of $M_{1}$ and $M_{2}$, respectively. However, the notation $M_{1}$ and $M_{2}$ has become more common.

The above conventions for the signs of the $\mu$ and the $A$ terms are the same as those adopted in ISAJET [2] and Haber and Kane [4]. This also agrees with the conventions of the other major SUSY event generators, SPYTHIA [7] and SUSYGEN [8]. While these conventions are the same internally, ISAJET uses a different notation which corresponds to the following:

$$
\begin{aligned}
2 m_{1} & =-\mu, \\
\mu_{1} & =-M_{1}, \\
\mu_{2} & =-M_{2},
\end{aligned}
$$

where $m_{1}, \mu_{1}$ and $\mu_{2}$ are in the notation of ISAJET.

\subsection{Mixing conventions}

While internally HERWIG uses the conventions of refs. [田, 6, the mass and decay spectra are fed in from a data file. In particular, we have provided a separate code (ISAWIG) for the conversion of data from ISAJET [2]. This way, the masses of the sparticles and their R-parity conserving decay rates are calculated using ISAJET and the R-parity violating section is added by ISAWIG. ISAJET also calculates the mixing matrices for the electroweak gauginos and scalar fermions. ISAJET uses the conventions of refs. [2, 10] and it is essential that the conversion between the two formalisms is done correctly.

We first consider the conversion between the two formalisms for the gauginos and then for the left-right sfermion mixing. We compare the lagrangian of ref. [2] against that of refs. [4, 5] to obtain the conversions between them. We adopt the conventions of refs. [⿴囗十 5 for the chargino and neutralino mixing matrices.

\subsubsection{Charginos}

In the notation of ref. [0], we define the following doublets of two-component spinors for the charginos in their interaction eigenstates:

$$
\begin{aligned}
& \psi^{+}=\left(-i \lambda^{+}, \psi_{H_{2}}^{+}\right), \\
& \psi^{-}=\left(-i \lambda^{-}, \psi_{H_{1}}^{-}\right),
\end{aligned}
$$

where $\lambda^{ \pm}$are the charged Winos, $\psi_{H_{1}}^{-}$is the charged Higgsino associated with the Higgs that gives mass to the down-type quarks and $\psi_{H_{2}}^{+}$is the charged Higgsino associated with 
the Higgs doublet that gives mass to the up-type quarks. The mass term in the lagrangian from ref. [5, eq. (A.2)] is:

$$
\mathcal{L}_{\text {chargino }}=-\frac{1}{2}\left(\psi^{+} \psi^{-}\right)\left(\begin{array}{cc}
0 & X^{T} \\
X & 0
\end{array}\right)\left(\begin{array}{l}
\psi^{+} \\
\psi^{-}
\end{array}\right)+(\text {h.c. })
$$

where the mass matrix is given by:

$$
X=\left(\begin{array}{cc}
M_{2} & M_{W} \sqrt{2} \sin \beta \\
M_{W} \sqrt{2} \cos \beta & \mu
\end{array}\right) .
$$

In ref. [5] this is diagonalized in the two-component notation by defining the mass eigenstates:

$$
\begin{aligned}
& \chi_{i}^{+}=V_{i j} \psi_{j}^{+}, \\
& \chi_{i}^{-}=U_{i j} \psi_{j}^{-},
\end{aligned}
$$

where $U$ and $V$ are unitary matrices chosen such that

$$
U^{*} X V^{-1}=M_{D}
$$

$M_{D}$ is the diagonal chargino mass matrix. The four-component mass eigenstates, the charginos, are defined in terms of the two-component fields as:

$$
\tilde{\chi}_{1}^{+}=\left(\begin{array}{c}
\chi_{1}^{+} \\
\left(\chi_{1}^{-}\right)^{c}
\end{array}\right), \quad \tilde{\chi}_{2}^{+}=\left(\begin{array}{c}
\chi_{2}^{+} \\
\left(\chi_{2}^{-}\right)^{c}
\end{array}\right) .
$$

We need to express the chargino mass terms in the lagrangian in a four-component notation so that we can compare this lagrangian with the conventions of ref. [2]. We define the fourcomponent spinors as in ref. [5]:

$$
\widetilde{W}^{+}=\left(\begin{array}{c}
-i \lambda^{+} \\
\left(-i \lambda^{-}\right)^{c}
\end{array}\right), \quad \widetilde{H}^{+}=\left(\begin{array}{c}
\psi_{H_{2}}^{+} \\
\left(\psi_{H_{1}}^{-}\right)^{c}
\end{array}\right) .
$$

Using this notation, we can express the lagrangian in the four-component notation:

$$
\mathcal{L}_{\text {chargino }}=-\left(\widetilde{\widetilde{W}^{+}} \widetilde{\widetilde{H}^{+}}\right)\left[X P_{L}+X^{T} P_{R}\right]\left(\begin{array}{c}
\widetilde{W}^{+} \\
\widetilde{H}^{+}
\end{array}\right) .
$$

The chirality projection operators are defined as $P_{L / R}=\left(1-/+\gamma_{5}\right) / 2$. Equation $(2.15)$ has a similar form to the chargino mass term ${ }^{2}$ in ref. [2, eq. (2.1)]:

$$
\mathcal{L}_{\text {chargino }}=-\left(\begin{array}{ll}
\bar{\lambda} & \bar{\chi}
\end{array}\right)\left[M_{\text {charge }} P_{L}+M_{\text {charge }}^{T} P_{R}\right]\left(\begin{array}{l}
\lambda \\
\chi
\end{array}\right)
$$

where

$$
M_{\text {charge }}=\left(\begin{array}{cc}
\mu_{2} & -g v^{\prime} \\
-g v & 2 m_{1}
\end{array}\right)=-\left(\begin{array}{cc}
M_{2} & \sqrt{2} M_{W} \cos \beta \\
\sqrt{2} M_{W} \sin \beta & \mu
\end{array}\right),
$$

\footnotetext{
${ }^{2}$ This lagrangian is taken from ref. [1]] which corrects a sign error in the off-diagonal terms in ref. [2].
} 
where $v$ and $v^{\prime}$ are the vacuum expectation values for the Higgs fields that give mass to the up- and down-type quarks, respectively. We now come to the problem of comparing the notation of ref. [5] against that of ref. [2]. The Wino and Higgsino fields of ref. [2 are the charge conjugates of those used in ref. [0], so that the following transformation is required:

$$
\begin{aligned}
\widetilde{W}^{+} & =\lambda^{c}, \\
\widetilde{H}^{+} & =\chi^{c} .
\end{aligned}
$$

This gives the chargino mass matrix in the form:

$$
\mathcal{L}_{\text {chargino }}=\left(\overline{\widetilde{W}^{+}}, \overline{\widetilde{H}^{+}}\right)\left[M_{\text {charge }}^{\prime} P_{L}+M_{\text {charge }}^{\prime T} P_{R}\right]\left(\begin{array}{c}
\widetilde{W}^{+} \\
\widetilde{H}^{+}
\end{array}\right)
$$

where

$$
M_{\text {charge }}^{\prime}=\left(\begin{array}{cc}
-\mu_{2} & g v \\
g v^{\prime} & -2 m_{1}
\end{array}\right)=\left(\begin{array}{cc}
M_{2} & \sqrt{2} M_{W} \sin \beta \\
\sqrt{2} M_{W} \cos \beta & \mu
\end{array}\right) .
$$

This agrees with eq. (2.15) apart from the overall sign. We need to express the fields in terms of the ISAJET mixing matrices. The ISAJET mixing matrices are given in ref. [2, eqs. (2.10) and (2.11)] as:

$$
\begin{array}{r}
\left(\begin{array}{c}
\widetilde{W}_{+} \\
\widetilde{W}_{-}
\end{array}\right)_{L}=\left(\begin{array}{cc}
\theta_{x} \cos \gamma_{L} & -\theta_{x} \sin \gamma_{L} \\
\sin \gamma_{L} & \cos \gamma_{L}
\end{array}\right)\left(\begin{array}{l}
\lambda \\
\chi
\end{array}\right)_{L} \\
\left(\begin{array}{c}
(-1)^{\theta+} \widetilde{W}_{+} \\
(-1)^{\theta-} \widetilde{W}_{-}
\end{array}\right)_{R}=\left(\begin{array}{cc}
\theta_{y} \cos \gamma_{R} & -\theta_{y} \sin \gamma_{R} \\
\sin \gamma_{R} & \cos \gamma_{R}
\end{array}\right)\left(\begin{array}{l}
\lambda \\
\chi
\end{array}\right)_{R} .
\end{array}
$$

The mixing angles $\gamma_{L}$ and $\gamma_{R}$, and the sign functions $\theta_{x}, \theta_{y}, \theta_{+}$, and $\theta_{-}$are defined in ref. 2].

We can transform these equations into the notation of ref. 14 with the identification:

$$
\begin{aligned}
& \widetilde{\chi}_{1}^{+}=\widetilde{W}_{-}^{c}, \\
& \widetilde{\chi}_{2}^{+}=\widetilde{W}_{+}^{c} .
\end{aligned}
$$

This gives:

$$
\begin{aligned}
& P_{L}\left(\begin{array}{c}
\widetilde{W}^{+} \\
\widetilde{H}^{+}
\end{array}\right)=P_{L}\left(\begin{array}{cc}
-\sin \gamma_{R} & -\theta_{y} \cos \gamma_{R} \\
-\cos \gamma_{R} & \theta_{y} \sin \gamma_{R}
\end{array}\right)\left(\begin{array}{c}
(-1)^{\theta_{-}} \widetilde{\chi}_{1}^{+} \\
(-1)^{\theta_{+}} \widetilde{\chi}_{2}^{+}
\end{array}\right), \\
& P_{R}\left(\begin{array}{c}
\widetilde{W}^{+} \\
\widetilde{H}^{+}
\end{array}\right)=P_{R}\left(\begin{array}{cc}
-\sin \gamma_{L} & -\theta_{x} \cos \gamma_{L} \\
-\cos \gamma_{L} & \theta_{x} \sin \gamma_{L}
\end{array}\right)\left(\begin{array}{c}
\widetilde{\chi}_{1}^{+} \\
\widetilde{\chi}_{2}^{+}
\end{array}\right) .
\end{aligned}
$$

These mixing matrices are now in a form that allows us to compare them with the notation of ref. [5, eq. (A.13)], :

$$
\begin{aligned}
& P_{L}\left(\begin{array}{c}
\widetilde{W}^{+} \\
\widetilde{H}^{+}
\end{array}\right)=P_{L}\left(\begin{array}{ll}
V_{11}^{*} & V_{21}^{*} \\
V_{12}^{*} & V_{22}^{*}
\end{array}\right)\left(\begin{array}{c}
\widetilde{\chi}_{1}^{+} \\
\widetilde{\chi}_{2}^{+}
\end{array}\right), \\
& P_{R}\left(\begin{array}{c}
\widetilde{W}^{+} \\
\widetilde{H}^{+}
\end{array}\right)=P_{R}\left(\begin{array}{ll}
U_{11} & U_{21} \\
U_{12} & U_{22}
\end{array}\right)\left(\begin{array}{l}
\widetilde{\chi}_{1}^{+} \\
\widetilde{\chi}_{2}^{+}
\end{array}\right) .
\end{aligned}
$$


By comparing eqs. (2.23) and (2.24) we obtain the mixing matrices in the notation of ref. [5]:

$$
\begin{aligned}
U & =\left(\begin{array}{cc}
-\sin \gamma_{L} & -\cos \gamma_{L} \\
-\theta_{x} \cos \gamma_{L} & \theta_{x} \sin \gamma_{L}
\end{array}\right) \\
V & =\left(\begin{array}{cc}
-\sin \gamma_{R}(-1)^{\theta_{-}} & -\cos \gamma_{R}(-1)^{\theta_{+}} \\
-\theta_{y} \cos \gamma_{R}(-1)^{\theta_{-}} & \theta_{y} \sin \gamma_{R}(-1)^{\theta_{+}}
\end{array}\right) .
\end{aligned}
$$

It should be noted that we adopt the opposite sign convention for the chargino masses due to the sign differences in the two lagrangians.

\subsubsection{Neutralinos}

We define a quadruplet of two-component fermion fields for the neutralinos in the interaction eigenstate as:

$$
\psi^{0}=\left(-i \lambda^{\prime},-i \lambda^{3}, \psi_{H_{1}}^{0}, \psi_{H_{2}}^{0}\right),
$$

where $\lambda^{\prime}$ is the Bino, $\lambda^{3}$ is the neutral Wino, $\psi_{H_{1}}^{0}$ is the Higgsino corresponding to the neutral component of the Higgs doublet giving mass to the down-type quarks and $\psi_{\mathrm{H}_{2}}^{0}$ is the Higgsino corresponding to the neutral component of the Higgs doublet giving mass to the up-type quarks. The lagrangian for the neutralino masses from ref. [5. eq. (A.18)] is:

$$
\left.\mathcal{L}_{\text {neutralino }}=-\frac{1}{2}\left(\psi^{0}\right)^{T} Y \psi^{0}+\text { (h.c. }\right)
$$

where

$$
\begin{aligned}
& Y= \\
& =\left(\begin{array}{cccc}
M_{1} & 0 & -M_{Z} \sin \theta_{W} \cos \beta & M_{Z} \sin \theta_{W} \sin \beta \\
0 & M_{2} & M_{Z} \cos \theta_{W} \cos \beta & -M_{Z} \cos \theta_{W} \sin \beta \\
-M_{Z} \sin \theta_{W} \cos \beta & M_{Z} \cos \theta_{W} \cos \beta & 0 & -\mu \\
M_{Z} \sin \theta_{W} \sin \beta & -M_{Z} \cos \theta_{W} \sin \beta & -\mu & 0
\end{array}\right) .
\end{aligned}
$$

In ref. [5], the lagrangian is diagonalized in this two-component notation to give the mass eigenstates. The diagonalization is performed by defining two-component fields:

$$
\chi_{i}^{0}=N_{i j} \psi_{j}^{0}, \quad i, j=1, \ldots, 4,
$$

where $N$ is a unitary matrix satisfying $N^{*} Y N^{-1}=N_{D}$, and $N_{D}$ is the diagonal neutralino mass matrix. The four-component mass eigenstates, the neutralinos, can be defined in terms of the two-component fields:

$$
\widetilde{\chi}_{i}^{0}=\left(\begin{array}{c}
\chi_{i}^{0} \\
\left(\chi_{i}^{0}\right)^{c}
\end{array}\right)
$$

Rather than adopting this approach we need to express the neutralino mass terms in the four-component notation before performing the diagonalization in order to compare this lagrangian with that of ref. [2]. We use the standard procedure of ref. [5] to express this 
in the four-component notation by defining four-component Majorana fields:

$$
\begin{array}{rlrl}
\widetilde{B} & =\left(\begin{array}{c}
-i \lambda^{\prime} \\
\left(-i \lambda^{\prime}\right)^{c}
\end{array}\right), & \widetilde{W}_{3}=\left(\begin{array}{c}
-i \lambda^{3} \\
\left(-i \lambda^{3}\right)^{c}
\end{array}\right), \\
\widetilde{H}_{1}=\left(\begin{array}{c}
\psi_{H_{1}}^{0} \\
\left(\psi_{H_{1}}^{0}\right)^{c}
\end{array}\right), & \widetilde{H}_{2}=\left(\begin{array}{c}
\psi_{H_{2}}^{0} \\
\left(\psi_{H_{2}}^{0}\right)^{c}
\end{array}\right) .
\end{array}
$$

As defined in table 2, $\widetilde{B}$ is the Bino field, $\widetilde{W}_{3}$ is the neutral Wino field, $\widetilde{H}_{1}$ is the field for the Higgsino associated with the neutral component of the Higgs doublet that gives mass to the down-type quarks and $\widetilde{H}_{2}$ is the field for the Higgsino associated with the neutral component of the Higgs doublet that gives mass to the up-type quarks.

This gives the lagrangian in the four-component notation:

$$
\mathcal{L}_{\text {neutralino }}=-\frac{1}{2}\left(\overline{\widetilde{B}} \overline{\widetilde{W}_{3}} \frac{\overline{H_{1}}}{\widetilde{H}_{2}}\right)\left[Y P_{L}+Y P_{R}\right]\left(\begin{array}{c}
\widetilde{B} \\
\widetilde{W}_{3} \\
\widetilde{H}_{1} \\
\widetilde{H}_{2}
\end{array}\right) .
$$

We can now compare this with the relevant lagrangian, in the four-component notation, given in ref. [2]. This lagrangian ${ }^{3}$ is from ref. [2, eq. (2.2)]:

$$
\mathcal{L}_{\text {neutralino }}=-\frac{1}{2}\left(\bar{h}^{0}, \bar{h}^{\prime}, \bar{\lambda}_{3}, \overline{\lambda_{0}}\right)\left[M_{\text {neutral }} P_{L}+M_{\text {neutral }} P_{R}\right]\left(\begin{array}{c}
h^{0} \\
h^{\prime 0} \\
\lambda_{3} \\
\lambda_{0}
\end{array}\right) .
$$

$h^{0}$ is the Higgsino partner of the neutral component of the Higgs doublet that gives mass to the up-type quarks, ${h^{\prime}}^{0}$ is the Higgsino partner of the Higgs boson that gives mass to the down-type quarks, $\lambda_{3}$ is the neutral Wino and $\lambda_{0}$ is the Bino. The mass matrix is given by:

$$
M_{\text {neutral }}=\left(\begin{array}{cccc}
0 & -2 m_{1} & -g v / \sqrt{2} & g^{\prime} v / \sqrt{2} \\
-2 m_{1} & 0 & g v^{\prime} / \sqrt{2} & -g^{\prime} v^{\prime} / \sqrt{2} \\
-g v / \sqrt{2} & g v^{\prime} / \sqrt{2} & \mu_{2} & 0 \\
g^{\prime} v / \sqrt{2} & -g^{\prime} v^{\prime} / \sqrt{2} & 0 & \mu_{1}
\end{array}\right) .
$$

It is easier to compare this with eq. (2.32) after reordering the entries and reexpressing it in terms of $M_{Z}, \beta$ and $\theta_{W}$. This gives:

$$
\mathcal{L}_{\text {neutralino }}=\frac{1}{2}\left(\bar{\lambda}_{0}, \bar{\lambda}_{3}, \bar{h}^{\prime 0}, \bar{h}^{0}\right)\left[M_{\text {neutral }}^{\prime} P_{L}+M_{\text {neutral }}^{\prime} P_{R}\right]\left(\begin{array}{c}
\lambda_{0} \\
\lambda_{3} \\
h^{\prime 0} \\
h^{0}
\end{array}\right),
$$

where:

$$
\begin{aligned}
& M_{\text {neutral }}^{\prime}= \\
& =\left(\begin{array}{cccc}
M_{1} & 0 & M_{Z} \sin \theta_{W} \cos \beta & -M_{Z} \sin \theta_{W} \sin \beta \\
0 & M_{2} & -M_{Z} \cos \theta_{W} \cos \beta & M_{Z} \cos \theta_{W} \sin \beta \\
M_{Z} \sin \theta_{W} \cos \beta & -M_{Z} \cos \theta_{W} \cos \beta & 0 & -\mu \\
-M_{Z} \sin \theta_{W} \sin \beta & M_{Z} \cos \theta_{W} \sin \beta & -\mu & 0
\end{array}\right) .
\end{aligned}
$$

\footnotetext{
${ }^{3}$ This lagrangian is taken from ref. [1]] which corrects a sign error in ref. [2].
} 
This equation then agrees with eq. (2.32) up to an overall sign, provided we make the following identification:

$$
\begin{aligned}
\widetilde{B} & =\lambda_{0}, & \widetilde{W}_{3}=\lambda_{3}, \\
\widetilde{H}_{1} & =-h^{\prime 0}, & \widetilde{H}_{2}=-h^{0} .
\end{aligned}
$$

The convention from ISAJET for the mixing, taken from ref. [2, eq. (2.12)], is

$$
\left(\begin{array}{ccc}
\left(-i \gamma_{5}\right)^{\theta_{1}} & \widetilde{Z}_{1} \\
\left(-i \gamma_{5}\right)^{\theta_{2}} & \widetilde{Z}_{2} \\
\left(-i \gamma_{5}\right)^{\theta_{3}} & \widetilde{Z}_{3} \\
\left(-i \gamma_{5}\right)^{\theta_{4}} & \widetilde{Z}_{4}
\end{array}\right)=\left(\begin{array}{cccc}
v_{1}^{(1)} & v_{2}^{(1)} & v_{3}^{(1)} & v_{4}^{(1)} \\
v_{1}^{(2)} & v_{2}^{(2)} & v_{3}^{(2)} & v_{4}^{(2)} \\
v_{1}^{(3)} & v_{2}^{(3)} & v_{3}^{(3)} & v_{4}^{(3)} \\
v_{1}^{(4)} & v_{2}^{(4)} & v_{3}^{(4)} & v_{4}^{(4)}
\end{array}\right)\left(\begin{array}{c}
h^{0} \\
h^{\prime 0} \\
\lambda_{3} \\
\lambda_{0}
\end{array}\right)
$$

where $\widetilde{Z}_{i}$ are the mass eigenstates obtained by diagonalizing the mass matrix given in eq. (2.34), $v_{j}^{(i)}$ are the elements of the mixing matrix, and $\theta_{i}$ is zero (one) if the mass of $\widetilde{Z}_{i}$ is positive (negative).

After reordering, we get:

$$
\left(\begin{array}{ccc}
\left(-i \gamma_{5}\right)^{\theta_{1}} & \widetilde{Z}_{1} \\
\left(-i \gamma_{5}\right)^{\theta_{2}} & \widetilde{Z}_{2} \\
\left(-i \gamma_{5}\right)^{\theta_{3}} & \widetilde{Z}_{3} \\
\left(-i \gamma_{5}\right)^{\theta_{4}} & \widetilde{Z}_{4}
\end{array}\right)=\left(\begin{array}{cccc}
v_{4}^{(1)} & v_{3}^{(1)} & -v_{2}^{(1)} & -v_{1}^{(1)} \\
v_{4}^{(2)} & v_{3}^{(2)} & -v_{2}^{(2)} & -v_{1}^{(2)} \\
v_{4}^{(3)} & v_{3}^{(3)} & -v_{2}^{(3)} & -v_{1}^{(3)} \\
v_{4}^{(4)} & v_{3}^{(4)} & -v_{2}^{(4)} & -v_{1}^{(4)}
\end{array}\right)\left(\begin{array}{c}
\widetilde{B} \\
\widetilde{W} \\
\widetilde{H}_{1} \\
\widetilde{H}_{2}
\end{array}\right)
$$

We can therefore obtain the mixing matrix in the notation of ref. [5 by making the identification:

$$
\begin{aligned}
& N_{i 1}=v_{4}^{(i)}, \quad N_{i 2}=v_{3}^{(i)}, \\
& N_{i 3}=-v_{2}^{(i)}, \quad N_{i 4}=-v_{1}^{(i)} .
\end{aligned}
$$

Again, we need to adopt the opposite sign convention for the neutralino masses.

\subsubsection{Left-right sfermion mixing}

In addition to the mixing of the neutralinos and charginos, we need to consider the leftright mixing of the sfermions. In general, as the off-diagonal terms in the mass matrices are proportional to the fermion mass, these effects are only important for the third generation sfermions, i.e. stop, sbottom and stau. We describe our convention for the top squarks. The others are treated analogously.

The following mass matrix for the top squarks uses the conventions ${ }^{4}$ of ref. [5] and is taken from eq. (4.17) therein.

$$
\begin{aligned}
M_{\widetilde{t}}^{2} & = \\
& =\left(\begin{array}{cc}
M_{\widetilde{Q}}^{2}+M_{Z}^{2} \cos 2 \beta\left(\frac{1}{2}-\frac{2}{3} \sin ^{2} \theta_{W}\right)+m_{t}^{2} & m_{t}\left(A_{t}-\mu \cot \beta\right) \\
m_{t}\left(A_{t}-\mu \cot \beta\right) & M_{\widetilde{U}}^{2}+\frac{2}{3} m_{Z}^{2} \cos 2 \beta \sin ^{2} \theta_{W}+m_{t}^{2}
\end{array}\right),
\end{aligned}
$$

where $M_{\widetilde{Q}}$ and $M_{\widetilde{U}}$ are soft SUSY-breaking masses for the left and right top squarks, respectively. $A_{t}$ is the trilinear soft SUSY-breaking term for the interaction of the left and right

\footnotetext{
${ }^{4}$ This is the same as ref. [12], SPYTHIA [7] and SUSYGEN [8].
} 
stop squarks with the Higgs boson. This compares with the ISAJET matrix from ref. [10]:

$$
\begin{aligned}
M_{\widetilde{t}}^{2} & = \\
& =\left(\begin{array}{cc}
M_{\widetilde{t}_{L}}^{2}+M_{Z}^{2} \cos 2 \beta\left(\frac{1}{2}-\frac{2}{3} \sin ^{2} \theta_{W}\right)+m_{t}^{2} & -m_{t}\left(A_{t}-\mu \cot \beta\right) \\
-m_{t}\left(A_{t}-\mu \cot \beta\right) & M_{\widetilde{t}_{R}}^{2}+\frac{2}{3} m_{Z}^{2} \cos 2 \beta \sin ^{2} \theta_{W}+m_{t}^{2}
\end{array}\right),
\end{aligned}
$$

where $M_{\widetilde{t}_{L}}^{2}=M_{\widetilde{Q}}^{2}$ and $M_{\widetilde{t}_{R}}^{2}=M_{\widetilde{U}}^{2}$. There is a difference in the sign of the off-diagonal terms. This means that, as the sign conventions for the $\mu$ and $A$ terms are the same, there is a difference in the relative phases of the two fields in the different conventions. Hence we should apply the following change to the ISAJET output:

$$
\theta_{t} \longrightarrow-\theta_{t}
$$

i.e. change the sign of the stop mixing angle. The same argument also applies to the sbottom and stau mixing angles.

We adopt the following convention for the sfermion mixing matrices:

$$
\left(\begin{array}{c}
\widetilde{q}_{i L} \\
\widetilde{q}_{i R}
\end{array}\right)=\left(\begin{array}{cc}
\cos \theta_{q}^{i} & \sin \theta_{q}^{i} \\
-\sin \theta_{q}^{i} & \sin \theta_{q}^{i}
\end{array}\right)\left(\begin{array}{c}
\widetilde{q}_{i 1} \\
\widetilde{q}_{i 2}
\end{array}\right)=\left(\begin{array}{cc}
Q_{L 1}^{i} & Q_{L 2}^{i} \\
Q_{R 1}^{i} & Q_{R 2}^{i}
\end{array}\right)\left(\begin{array}{c}
\widetilde{q}_{i 1} \\
\widetilde{q}_{i 2}
\end{array}\right),
$$

where $\widetilde{q}_{i L}$ and $\widetilde{q}_{i R}$ are the left and right squark fields, for the $i$ th quark, where $i$ runs over quark flavours $d, u, s, c, b, t . \widetilde{q}_{i 1}$ and $\widetilde{q}_{i 2}$ are the squark fields for the mass eigenstates, for the quark $i$, and $\theta_{q}^{i}$ is the mixing angle obtained by diagonalizing the mass matrix.

Similarly, we denote the slepton mixing as above with the matrix $L_{\alpha \beta}^{i}$ where $i$ runs over the charged and neutral lepton flavour. $\beta$ is the mass eigenstate and $\alpha$ is the left/right eigenstate. As we do not include the right-handed neutrino we omit the left-right mixing for the sneutrinos. Note that our convention for the mixing matrix is opposite to that for the gauginos, in the sense that here the mixing matrices $Q^{i}$ act on mass eigenstates to give the interaction eigenstates.

\subsubsection{Higgs bosons}

The Higgs doublets are diagonalised as follows. As in tables 1 and 2 $H_{i}$ denote the $\mathrm{SU}(2)$ doublet interaction eigenstates which form the physical eigenstates $h^{0}, H^{0}, A^{0}, H^{ \pm}$after the electroweak symmetry breaking. In the charged sector:

$$
H_{2}^{+}=H^{+} \cos \beta+G^{+} \sin \beta, \quad H_{1}^{-}=H^{-} \sin \beta-G^{-} \cos \beta,
$$

where $G^{ \pm}$are the Goldstone modes. The neutral Higgs sector is parametrised in the interaction eigenstate as follows:

$$
\begin{aligned}
& H_{1}^{0}=\frac{v \cos \beta+\eta_{1}+i \zeta_{1}}{\sqrt{2}}, \\
& H_{2}^{0}=\frac{v \sin \beta+\eta_{2}+i \zeta_{2}}{\sqrt{2}} .
\end{aligned}
$$

$v$ is the vacuum expectation value. The diagonalisation is achieved by:

$$
\begin{array}{ll}
\zeta_{2}=A^{0} \cos \beta+G^{0} \sin \beta, & \zeta_{1}=A^{0} \sin \beta-G^{0} \cos \beta, \\
\eta_{2}=H^{0} \sin \alpha+h^{0} \cos \alpha, & \eta_{1}=H^{0} \cos \alpha-h^{0} \sin \alpha .
\end{array}
$$

Our notation is consistent with ref. [5], but the sign of $\alpha$ is opposite to that of ISAJET [2. 


\section{Matrix elements}

\subsection{Notation}

For $2 \rightarrow 2$ hard scattering subprocesses of the form $12 \rightarrow 34$, let us define the usual Mandelstam variables in terms of the partonic momenta as $\hat{s}=\left(p_{1}+p_{2}\right)^{2}, \hat{t}=\left(p_{1}-p_{3}\right)^{2}$ and $\hat{u}=\left(p_{1}-p_{4}\right)^{2}$. For simplicity, the "hat" will be omitted in the following. As initial state partons are taken to have zero kinematic mass, it follows that $s+t+u=m_{3}^{2}+m_{4}^{2}$, $s+t_{3}+u_{4}=0$, and $u t-m_{3}^{2} m_{4}^{2}=s p_{T}^{2}$ where $t_{3}=t-m_{3}^{2}, u_{4}=u-m_{4}^{2}$, and $p_{T}$ is the outgoing transverse momentum.

The electromagnetic coupling is $e$, such that $\alpha_{\mathrm{EM}}=e^{2} / 4 \pi$. The strong coupling is $g_{s}$, such that $\alpha_{s}=g_{s}^{2} / 4 \pi$. The QCD colour factors are $N_{C}=3$ and $C_{F}=\left(N_{C}^{2}-1\right) / 2 N_{C}=4 / 3$.

Colour flow directions are not stated explicitly as they are trivial in most of the processes whose matrix elements are given below. For a general treatment, see ref. [13]. Matrix elements given here are summed over the final state colour and spin, and averaged over the initial state colour and spin. As usual, this is indicated by a long overline. Statistical factors of two for identical neutralinos in the final state are written explicitly.

Let us now consider the widths $\Gamma\left(q^{2}\right)$ appearing in the propagators of unstable massive particles with mass $M\left(q^{2}\right)$. In order to simplify the notation, let us define a function $\bar{\Gamma}\left(q^{2}\right)$, which is defined for positive $q^{2}$ by:

$$
\sqrt{q^{2}} \Gamma\left(q^{2}\right) \equiv M\left(q^{2}\right) \bar{\Gamma}\left(q^{2}\right)
$$

For negative $q^{2}$, the width is taken to be zero. The propagator is then given in general, again for positive $q^{2}$, by:

$$
\frac{1}{q^{2}-M^{2}\left(q^{2}\right)+i \sqrt{q^{2}} \Gamma\left(q^{2}\right)} \equiv \frac{1}{q^{2}-M^{2}\left(q^{2}\right)+i M\left(q^{2}\right) \bar{\Gamma}\left(q^{2}\right)} .
$$

In the HERWIG implementation, we adopt the naive running width approximation and neglect the running of mass, so that:

$$
\bar{\Gamma}\left(q^{2}\right)=\frac{q^{2} \Gamma\left(M^{2}\right)}{M^{2}} .
$$

\begin{tabular}{|c|c|c|}
\hline$f$ & $Q_{f}$ & $I_{f}^{3}$ \\
\hline$e$ & -1 & $-1 / 2$ \\
$\nu$ & 0 & $+1 / 2$ \\
$d$ & $-1 / 3$ & $-1 / 2$ \\
$u$ & $+2 / 3$ & $+1 / 2$ \\
\hline
\end{tabular}

Table 3: The $U(1)_{\mathrm{EM}}$ charge $Q_{f}$ and the weak isospin $I_{f}^{3}$ for the Standard Model fermions.

The following coupling notations are used in processes involving electroweak interactions:

$$
\begin{aligned}
Q_{f} & =U(1)_{\mathrm{EM}} \text { charge } ; \\
I_{f}^{3} & =\text { weak isospin } ; \\
L_{f} & =\frac{\left(I_{f}^{3}-Q_{f} \sin ^{2} \theta_{W}\right)}{\cos \theta_{W} \sin \theta_{W}} ; \\
R_{f} & =\frac{-Q_{f} \sin ^{2} \theta_{W}}{\cos \theta_{W} \sin \theta_{W}} .
\end{aligned}
$$

$Q_{f}$ and $I_{f}^{3}$ are as listed in table 3. In terms of the above, we define the neutralino to 
sfermion coupling coefficients as:

$$
\begin{aligned}
\widetilde{L}_{f a} & =\left(N_{a 1} \cos \theta_{W}+N_{a 2} \sin \theta_{W}\right) Q_{f}+\left(-N_{a 1} \sin \theta_{W}+N_{a 2} \cos \theta_{W}\right) L_{f} \\
\widetilde{R}_{f a} & =\left(N_{a 1} \cos \theta_{W}+N_{a 2} \sin \theta_{W}\right) Q_{f}+\left(-N_{a 1} \sin \theta_{W}+N_{a 2} \cos \theta_{W}\right) R_{f} .
\end{aligned}
$$

Following ref. [4], we have neglected the contribution of the Higgsino component, which is proportional to the Yukawa coupling. This affects the cross sections for sfermion-Higgsino associated production, namely the Higgsino component of the processes $g b \rightarrow \widetilde{t} \widetilde{\chi}^{ \pm}, \widetilde{b} \widetilde{\chi}^{0}$.

\subsection{Sparton pair production}

The matrix elements and the colour decompositions for $2 \rightarrow 2$ parton-to-sparton scattering are given in ref. [13]. The term "sparton" refers to coloured superparticles, namely the squarks and the gluino. Neglecting the squark left-right mixings, the lepton-annihilation reaction $\ell^{-} \ell^{+} \rightarrow \widetilde{q} \widetilde{q}^{*}$ is given by:

$$
\begin{aligned}
\overline{|\mathcal{M}|^{2}}(L, R)= & e^{4} N_{C} s p_{T}^{2} \times \\
& \times\left[\left|\frac{Q_{\ell} Q_{q}}{s}+\frac{L_{\ell}\left(L_{q}, R_{q}\right)}{s-M_{Z}^{2}+i M_{Z} \bar{\Gamma}_{Z}}\right|^{2}+\left|\frac{Q_{\ell} Q_{q}}{s}+\frac{R_{\ell}\left(L_{q}, R_{q}\right)}{s-M_{Z}^{2}+i M_{Z} \bar{\Gamma}_{Z}}\right|^{2}\right] .
\end{aligned}
$$

The generalization to the case with left-right mixings is given by:

$$
\left(L_{q}, R_{q}\right) \longrightarrow L_{q} Q_{L i} Q_{L j}+R_{q} Q_{R i} Q_{R j} .
$$

The indices $i, j$ label the squarks that are produced.

\subsection{Slepton pair production}

The matrix elements are similar to eqs. (3.9) and (3.10). For the quark annihilation reaction $q \bar{q} \rightarrow \widetilde{\ell \ell^{*}}$ one should interchange the indices $(q \leftrightarrow \ell)$, and change the colour factor from $N_{C}$ to $1 / N_{C}$. In addition, for the charged current reaction $q \bar{q}^{\prime} \rightarrow \widetilde{\ell}_{L} \widetilde{\nu}^{*}$ we have

$$
\overline{|\mathcal{M}|^{2}}\left(q \bar{q}^{\prime} \rightarrow \widetilde{\ell}_{L} \widetilde{\nu}^{*}\right)=\frac{e^{4} s p_{T}^{2}}{\left(2 \sin ^{2} \theta_{W}\right)^{2} N_{C}}\left|\frac{1}{s-M_{W}^{2}+i M_{W} \bar{\Gamma}_{W}}\right|^{2} .
$$

For the lepton annihilation reaction the colour factor is 1 , and there is an extra $t$-channel gaugino contribution to the production of slepton pairs of the same generation as the colliding lepton pair, namely,

$$
\begin{aligned}
& \overline{|\mathcal{M}|^{2}}\left(e^{-} e^{+} \rightarrow \widetilde{e}_{L} \widetilde{e}_{L}^{*}, \widetilde{e}_{R} \widetilde{e}_{R}^{*}\right)= e^{4} s p_{T}^{2} \times \\
& \times {\left[\left|\frac{1}{s}+\frac{L_{e}\left(L_{e}, R_{e}\right)}{s-M_{Z}^{2}+i M_{Z} \bar{\Gamma}_{Z}}+\sum_{a=1}^{4}\left(\frac{\widetilde{L}_{e a}^{2}}{t-M_{\widetilde{\chi}_{a}^{0}}^{2}}, 0\right)\right|^{2}+\right.} \\
&\left.+\left|\frac{1}{s}+\frac{R_{e}\left(L_{e}, R_{e}\right)}{s-M_{Z}^{2}+i M_{Z} \bar{\Gamma}_{Z}}+\sum_{a=1}^{4}\left(0, \frac{\widetilde{R}_{e a}^{2}}{t-M_{\widetilde{\chi}_{a}^{0}}^{2}}\right)\right|^{2}\right], \\
& \overline{|\mathcal{M}|^{2}}\left(e^{-} e^{+} \rightarrow \widetilde{e}_{L} \widetilde{e}_{R}^{*}, \widetilde{e}_{R} \widetilde{e}_{L}^{*}\right)=e^{4}\left(\sum_{a=1}^{4} \frac{\left.M_{\widetilde{\chi}_{a}^{0} \sqrt{s}} \widetilde{L}_{e a} \widetilde{R}_{e a}\right)^{2} .}{M_{\widetilde{\chi}_{a}^{0}}^{2}}\right.
\end{aligned}
$$


The neutralino masses appearing above are the signed masses. The production of sneutrinos of the same generation as the colliding lepton pair is given by:

$$
\begin{aligned}
\overline{|\mathcal{M}|^{2}}\left(e^{-} e^{+} \rightarrow \widetilde{\nu}_{e L} \widetilde{\nu}_{e L}^{*}\right)=e^{4} s p_{T}^{2} & {\left[\left|\frac{L_{e} L_{\nu}}{s-M_{Z}^{2}+i M_{Z} \bar{\Gamma}_{Z}}+\sum_{a=1}^{2} \frac{\left(V_{a 1}^{\widetilde{\chi}^{ \pm}}\right)^{2} / 2 \sin ^{2} \theta_{W}}{t-M_{\widetilde{\chi}_{a}^{ \pm}}^{2}}\right|^{2}+\right.} \\
& \left.+\left|\frac{R_{e} L_{\nu}}{s-M_{Z}^{2}+i M_{Z} \bar{\Gamma}_{Z}}\right|^{2}\right]
\end{aligned}
$$

\subsection{Gaugino production}

We consider the following seven processes in hadronic collisions:

$$
\begin{aligned}
q \bar{q} \longrightarrow \widetilde{\chi}_{a}^{+} \widetilde{\chi}_{b}^{-}, \\
q \bar{q} \longrightarrow \widetilde{\chi}_{i}^{0} \widetilde{\chi}_{j}^{0}, \\
q \bar{q}^{\prime} \longrightarrow \widetilde{\chi}_{a}^{ \pm} \widetilde{\chi}_{i}^{0}, \\
q \bar{q} \longrightarrow \widetilde{\chi}_{i}^{0} \widetilde{g}, \\
g q \longrightarrow \widetilde{\chi}_{i}^{0} \widetilde{q} \quad \text { and c.c. } \\
q \bar{q}^{\prime} \longrightarrow \widetilde{\chi}_{a}^{ \pm} \widetilde{g}, \\
g q \longrightarrow \widetilde{\chi}_{a}^{ \pm} \widetilde{q}^{\prime} \quad \text { and c.c. }
\end{aligned}
$$

c.c. refers to charge conjugation. The indices take the values $a, b=1,2$ and $i, j=1, \ldots, 4$. Only processes (3.15) and (3.16) are relevant to leptonic collisions, where the colour factors and electroweak coupling coefficient need trivial modifications. Processes (3.15), (3.16), (3.17), (3.18) and (3.20) are expressed conveniently following the notation of ref. [14]:

$$
A=\sum_{\alpha, \beta=L, R} C_{\alpha \beta}\left(\bar{v}_{2} \gamma^{\mu} P_{\alpha} u_{1}\right)\left(\bar{u}_{3} \gamma^{\mu} P_{\beta} v_{4}\right)=\sum_{\alpha, \beta=L, R} C_{\alpha \beta} Q_{\alpha}^{\mu} X_{\beta}^{\mu}
$$

where, as before, $P_{L}=\left(1-\gamma_{5}\right) / 2$ and $P_{R}=\left(1+\gamma_{5}\right) / 2$. The spin-averaged amplitudes are given as:

$$
\begin{aligned}
\overline{|A|^{2}}= & {\left[\left|C_{L L}\right|^{2}+\left|C_{R R}\right|^{2}\right] u_{3} u_{4}+\left[\left|C_{L R}\right|^{2}+\left|C_{R L}\right|^{2}\right] t_{3} t_{4}+} \\
& +2 \operatorname{Re}\left[C_{L L}^{*} C_{L R}+C_{R R}^{*} C_{R L}\right]\left(m_{3} m_{4} s\right) .
\end{aligned}
$$

The masses appearing above as $\left(m_{3} m_{4} s\right)$ are the signed masses.

The matrix elements squared for processes (3.19) and (3.21) have the following form, where $\alpha$ labels the chirality of the final state squark:

$$
\overline{|\mathcal{M}|^{2}}{ }_{\alpha}=\frac{\left(g_{s} g_{\alpha}\right)^{2}}{2 N_{C}}\left[-\frac{t_{3}}{s}-\frac{2\left(m_{4}^{2}-m_{3}^{2}\right) t_{3}}{s u_{4}}\left(1+\frac{m_{3}^{2}}{t_{3}}+\frac{m_{4}^{2}}{u_{4}}\right)\right] .
$$

When left-right squark mixing is considered, the above is modified as:

$$
\overline{|\mathcal{M}|^{2}}{ }_{\alpha} \propto * g_{\alpha}^{2} \longrightarrow \overline{|\mathcal{M}|^{2}}{ }_{i} \propto g_{i}^{2}, \quad \text { where } g_{i}^{2}=g_{L}^{2} Q_{L i}^{2}+g_{R}^{2} Q_{R i}^{2} .
$$


Process (3.15), $q \bar{q} \rightarrow \widetilde{\chi}_{a}^{+} \widetilde{\chi}_{b}^{-}$, is given by eq. (3.23) with the prefactor $\left(e^{4} / N_{C}\right)$ and the following coefficients $C_{\alpha \beta}$ :

$$
\begin{aligned}
C_{\alpha \beta}= & -\delta_{a b} \frac{Q_{q}}{s}-\frac{1}{\sin 2 \theta_{W}} \cdot \frac{\left(\delta_{\alpha L} L_{q}+\delta_{\alpha R} R_{q}\right)\left(-2 O_{a b}^{\prime \beta}\right)}{s-M_{Z}^{2}+i M_{Z} \bar{\Gamma}_{Z}}- \\
& -\frac{\delta_{\alpha L}}{2 \sin ^{2} \theta_{W}}\left[\frac{\left(1-\delta_{\alpha \beta}\right) \delta_{q u} U_{a 1} U_{b 1}}{t-M_{\widetilde{d}_{L}}^{2}}-\frac{\delta_{\alpha \beta} \delta_{q d} V_{a 1} V_{b 1}}{u-M_{\widetilde{u}_{L}}^{2}}\right] .
\end{aligned}
$$

Here, following the notation of ref. 四, we have defined:

$$
\begin{aligned}
& -2 O_{a b}^{\prime L}=-V_{a 2} V_{b 2}+2 \delta_{a b} \cos ^{2} \theta_{W} ; \\
& -2 O_{a b}^{\prime R}=-U_{a 2} U_{b 2}+2 \delta_{a b} \cos ^{2} \theta_{W} .
\end{aligned}
$$

Process (3.16), $q \bar{q} \rightarrow \widetilde{\chi}_{i}^{0} \widetilde{\chi}_{j}^{0}$, is given by eq. (3.22) with the prefactors $\left(e^{4} / N_{C}\right)$ and $1 /\left(1+\delta_{i j}\right)$, and the following coefficients $C_{\alpha \beta}$ :

$$
\begin{aligned}
C_{L L} & =\frac{L_{q}}{\sin 2 \theta_{W}} \cdot \frac{N_{i 4} N_{j 4}-N_{i 3} N_{j 3}}{s-M_{Z}^{2}+i M_{Z} \bar{\Gamma}_{Z}}+\frac{\widetilde{L}_{q i} \widetilde{L}_{q j}}{u-M_{\widetilde{q}_{L}}^{2}} ; \\
C_{L R} & =\frac{L_{q}}{\sin 2 \theta_{W}} \cdot \frac{N_{i 3} N_{j 3}-N_{i 4} N_{j 4}}{s-M_{Z}^{2}+i M_{Z} \bar{\Gamma}_{Z}}-\frac{\widetilde{L}_{q i} \widetilde{L}_{q j}}{t-M_{\widetilde{q}_{L}}^{2}} ; \\
C_{R L} & =\frac{R_{q}}{\sin 2 \theta_{W}} \cdot \frac{N_{i 4} N_{j 4}-N_{i 3} N_{j 3}}{s-M_{Z}^{2}+i M_{Z} \bar{\Gamma}_{Z}}-\frac{\widetilde{R}_{q i} \widetilde{R}_{q j}}{t-M_{\widetilde{q}_{R}}^{2}} ; \\
C_{R R} & =\frac{R_{q}}{\sin 2 \theta_{W}} \cdot \frac{N_{i 3} N_{j 3}-N_{i 4} N_{j 4}}{s-M_{Z}^{2}+i M_{Z} \bar{\Gamma}_{Z}}+\frac{\widetilde{R}_{q i} \widetilde{R}_{q j}}{u-M_{\widetilde{q}_{R}}^{2}} .
\end{aligned}
$$

Process (3.17), $u \bar{d} \rightarrow \widetilde{\chi}_{a}^{+} \widetilde{\chi}_{i}^{0}$, is given by eq. (3.22). The prefactors are given by $\left(e^{4} / N_{C}\right)$ and $\left(\left|V_{\mathrm{CKM}}\right|^{2} / 2 \sin ^{2} \theta_{W}\right)$ where $V_{\mathrm{CKM}}$ refers to the relevant term of the Cabbibo-KobayashiMaskawa (CKM) matrix, and the coefficients $C_{\alpha \beta}$ are:

$$
\begin{aligned}
C_{L L} & =\frac{1}{\sin \theta_{W}} \cdot \frac{N_{i 2} V_{a 1}-N_{i 4} V_{a 2} / \sqrt{2}}{s-M_{W}^{2}+i M_{W} \bar{\Gamma}_{W}}+\frac{V_{a 1} \widetilde{L}_{u i}}{u-M_{\widetilde{u}_{L}}^{2}} ; \\
C_{L R} & =\frac{1}{\sin \theta_{W}} \cdot \frac{N_{i 2} U_{a 1}+N_{i 3} U_{a 2} / \sqrt{2}}{s-M_{W}^{2}+i M_{W} \bar{\Gamma}_{W}}-\frac{U_{a 1} \widetilde{L}_{d i}}{t-M_{\widetilde{d}_{L}}^{2}} ; \\
C_{R L} & =0 ; \\
C_{R R} & =0 .
\end{aligned}
$$

Process (3.18), $q \bar{q} \rightarrow \widetilde{\chi}_{i}^{0} \widetilde{g}$, is given by eq. (3.22) with the prefactor $\left(e^{2} g_{s}^{2} C_{F} / N_{C}\right)$ and the following coefficients $C_{\alpha \beta}$ :

$$
\begin{aligned}
C_{L L} & =\frac{\widetilde{L}_{q i}}{u-M_{\widetilde{q}_{L}}^{2}} ; & C_{L R} & =-\frac{\widetilde{L}_{q i}}{t-M_{\widetilde{q}_{L}}^{2}} ; \\
C_{R L} & =\frac{\widetilde{R}_{q i}}{t-M_{\widetilde{q}_{R}}^{2}} ; & C_{R R} & =-\frac{\widetilde{R}_{q i}}{u-M_{\widetilde{q}_{R}}^{2}} .
\end{aligned}
$$


Process (3.19), $g q \rightarrow \widetilde{\chi}_{i}^{0} \widetilde{q}$, is given by eq. (3.24), with $g_{\alpha}=e\left(\delta_{\alpha L} \widetilde{L}_{q i}-\delta_{\alpha R} \widetilde{R}_{q i}\right)$.

Process (3.20), $u \bar{d} \rightarrow \tilde{\chi}_{a}^{+} \widetilde{g}$, is given by eq. (3.22). The prefactors are $\left(e^{2} g_{s}^{2} C_{F} / N_{C}\right)$ and $\left(\left|V_{\mathrm{CKM}}\right|^{2} / 2 \sin ^{2} \theta_{W}\right)$, and the coefficients $C_{\alpha \beta}$ are:

$$
\begin{aligned}
& C_{L L}=\frac{V_{a 1}}{u-M_{\widetilde{u}_{L}}^{2}} ; \quad C_{L R}=-\frac{U_{a 1}}{t-M_{\widetilde{d}_{L}}^{2}} ; \\
& C_{R L}=0 ; \quad C_{R R}=0 .
\end{aligned}
$$

Process (3.21), $g q \rightarrow \widetilde{\chi}_{a}^{ \pm} \widetilde{q}_{L}^{\prime}$, is given by eq. (3.24), with $g_{L}=e\left(\delta_{q u} U_{a 1}+\delta_{q d} V_{a 1}\right)$.

Processes (3.17), (3.20) and (3.21) involve flavour-changing CKM matrix contributions. We assume that the CKM matrix is universal and applicable also to sfermion interactions.

Process (3.15) involves two flavour-changing vertex contributions and, in principle, we should sum over the internal squark flavours and external quark pairs. However, we believe that to a good approximation this process can be treated correctly by substituting an identity matrix for the CKM matrix and so this is done in HERWIG.

\subsection{Neutral Higgs production}

For hadronic collisions, the following five classes of production subprocesses are implemented:

(a) gluon-gluon fusion via loops of heavy quarks $Q=b$ and $t$ and squarks $\widetilde{Q}=\widetilde{b}$ and $\widetilde{t}$,

(b) $W^{ \pm} W^{\mp}, Z^{0} Z^{0}$ fusion,

(c) associated production with $W^{ \pm}, Z^{0}$ bosons,

(d) associated production with pairs of heavy quarks $Q \bar{Q}$,

(e) associated production with pairs of heavy squarks $\widetilde{Q} \widetilde{Q}^{*}$.

The scalar Higgs bosons $h^{0}$ and $H^{0}$ can be produced by all five subprocesses (a)-(e), whereas the pseudoscalar $A^{0}$ is only produced via the first and the last two, as the $A^{0} V V$ vertex, with $V=W^{ \pm}, Z^{0}$, is absent at tree level. Higgs bosons are also produced in decays of heavier sparticles.

For leptonic collisions, the following are implemented:

(f) $W^{ \pm} W^{\mp}, Z^{0} Z^{0}$ fusion,

(g) associated production with $Z^{0}$,

(h) $h^{0} A^{0}$ and $H^{0} A^{0}$ production.

Of these, (f) and (g) are expressed by formulae that are obtained by changing the $Z^{0}$ boson couplings in the corresponding hadronic subprocesses. In addition, for $(\mathrm{g})$, the colour factor $1 / N_{C}$ is replaced by 1 . 


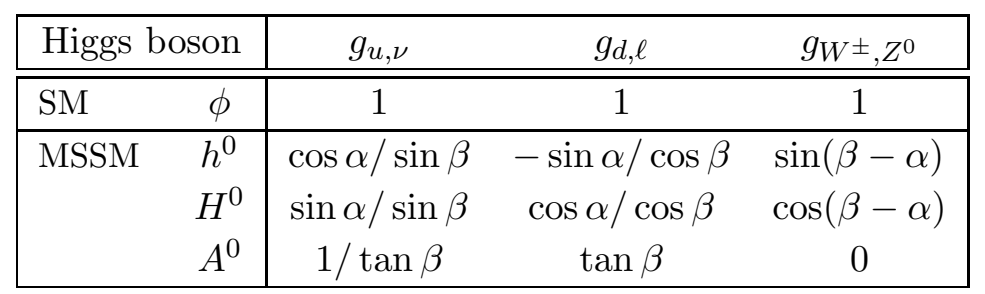

Table 4: Higgs coupling coefficients in the MSSM to isospin $+1 / 2$ and $-1 / 2$ fermions and to the gauge bosons $W^{ \pm}, Z^{0}$, as first appearing in eqs. (3.32) and (3.33).

In the following, let us denote the neutral Higgs bosons collectively by $\Phi$. We define the following notation for invariant mass squared. $M_{[a b]}^{2}$ is the invariant mass squared of the particle pair $[a b], M_{\left[a_{i} b_{i}\right]}^{2}=M_{[12]}^{2}=\left(p_{a}+p_{b}\right)^{2}=s, M_{\left[a_{f} b_{f}\right]}^{2}=\left(p_{a}+p_{b}\right)^{2}$ and $M_{\left[a_{i} b_{f}\right]}^{2}=\left(p_{a}-p_{b}\right)^{2}$, where the subscripts $i$ and $f$ indicate particles in the initial and final states, respectively. $M_{[a b]}^{2}$ is negative only in the last of these three cases.

For the gluon-fusion subprocess class (a), the matrix elements are cast in the form seen in ref. 15:

$$
\begin{aligned}
\overline{|\mathcal{M}|^{2}}\left(g g \rightarrow h^{0}, H^{0}\right)= & \frac{\alpha_{\mathrm{EM}} \alpha_{\mathrm{S}}^{2} M_{h, H}^{4}}{72 \pi \sin ^{2} \theta_{W}\left(N_{C}^{2}-1\right) M_{W}^{2}} \times \\
& \times\left|\sum_{f} g_{f}^{h, H} A_{f}^{h, H}\left(\tau_{f}\right)+\sum_{\widetilde{f}} g_{\widetilde{f}}^{h, H} A_{\widetilde{f}}^{h, H}\left(\tau_{\widetilde{f}}\right)\right|^{2}, \\
\overline{|\mathcal{M}|^{2}}\left(g g \rightarrow A^{0}\right)= & \frac{\alpha_{\mathrm{EM}} \alpha_{\mathrm{S}}^{2} M_{A}^{4}}{72 \pi \sin ^{2} \theta_{W}\left(N_{C}^{2}-1\right) M_{W}^{2}}\left|\sum_{f} g_{f}^{A} A_{f}^{A}\left(\tau_{f}\right)\right|^{2},
\end{aligned}
$$

for scalar and pseudoscalar production, respectively. The couplings $g_{f}$ and $g_{\tilde{f}}$ are those appearing in tables 4 and 5 , respectively. We have defined $\tau=4 m^{2} / M^{2}$, where $M$ is the mass of the Higgs boson concerned and $m$ is the mass of the particle inside the loop. The functions $A$ are given by:

$$
\begin{aligned}
& A_{Q}^{h, H}(\tau)=\frac{3}{2} \tau[1+(1-\tau) f(\tau)], \\
& A_{\widetilde{Q}}^{h, H}(\tau)=-\frac{3}{4}[1-\tau f(\tau)]
\end{aligned}
$$

for the scalars and

$$
A_{Q}^{A}(\tau)=\tau f(\tau),
$$

for the pseudoscalar, with the function $f(\tau)$ given by

$$
f(\tau)= \begin{cases}\arcsin ^{2} \frac{1}{\sqrt{\tau}}, & \tau \geq 1 \\ -\frac{1}{4}\left[\log \frac{1+\sqrt{1-\tau}}{1-\sqrt{1-\tau}}-i \pi\right]^{2}, & \tau<1\end{cases}
$$




\begin{tabular}{|c|c|}
\hline Higgs boson & $g_{\widetilde{f}_{i}}$ \\
\hline SM & 0 \\
\hline MSSM $\quad h^{0}$ & $\begin{array}{c}m_{\tilde{f}_{i}}^{-2}\left[m_{f}^{2} g_{f}^{h}+M_{Z}^{2} \cos \theta_{W} \sin \theta_{W}\left(L_{f} Q_{L i}^{2}-R_{f} Q_{R i}^{2}\right) \sin (\alpha+\beta)\right. \\
\left.+\left(\mu m_{f}\left(\delta_{f u} \frac{\sin \alpha}{\sin \beta}-\delta_{f d} \frac{\cos \alpha}{\cos \beta}\right)+A_{f} m_{f} g_{f}^{h}\right) Q_{L i} Q_{R i}\right]\end{array}$ \\
\hline$H^{0}$ & $\begin{aligned} m_{\tilde{f}_{i}}^{-2} & {\left[m_{f}^{2} g_{f}^{H}+M_{Z}^{2} \cos \theta_{W} \sin \theta_{W}\left(R_{f} Q_{R i}^{2}-L_{f} Q_{L i}^{2}\right) \cos (\alpha+\beta)\right.} \\
& \left.+\left(\mu m_{f}\left(-\delta_{f u} \frac{\cos \alpha}{\sin \beta}-\delta_{f d} \frac{\sin \alpha}{\cos \beta}\right)+A_{f} m_{f} g_{f}^{H}\right) Q_{L i} Q_{R i}\right]\end{aligned}$ \\
\hline$A^{0}$ & 0 \\
\hline
\end{tabular}

Table 5: MSSM Higgs coupling coefficients to sfermions $\tilde{f}=\tilde{q}, \tilde{\ell}$, as first appearing in eq. (3.32). For the leptonic case, the mixing matrices $Q_{i j}$ should be replaced by the corresponding slepton mixing matrices $L_{i j}$.

For process (b), the matrix element for the $W^{ \pm} W^{\mp}$ fusion process is given by:

$$
\begin{aligned}
\overline{|\mathcal{M}|^{2}}\left(q_{1} q_{2}^{\prime} \rightarrow q_{3}^{\prime \prime} q_{4}^{\prime \prime \prime} \Phi_{5}\right)= & \left(g_{W^{ \pm}}^{\Phi}\right)^{2}\left(\frac{e^{2}}{2 \sin ^{2} \theta_{W}}\right)^{3}\left(2 M_{W}^{2}\right) \times \\
& \times \frac{\left|V_{\mathrm{CKM}}\left[q q^{\prime \prime}\right]\right|^{2}\left|V_{\mathrm{CKM}}\left[q^{\prime} q^{\prime \prime \prime}\right]\right|^{2} M_{[12]}^{2} M_{[34]}^{2}}{\left(M_{[13]}^{2}-M_{W}^{2}\right)\left(M_{[24]}^{2}-M_{W}^{2}\right)},
\end{aligned}
$$

whereas for $Z^{0} Z^{0}$ fusion we have:

$$
\begin{aligned}
\overline{|\mathcal{M}|^{2}}\left(q_{1} q_{2}^{\prime} \rightarrow q_{3} q_{4}^{\prime} \Phi_{5}\right)= & \left(g_{Z^{0}}^{\Phi}\right)^{2}\left(\frac{e^{2}}{4 \sin ^{2} \theta_{W} \cos ^{2} \theta_{W}}\right)^{3}\left(4 M_{Z}^{2}\right) \times \\
& \times \frac{\left(L_{q}^{2} L_{q^{\prime}}^{2}+R_{q}^{2} R_{q^{\prime}}^{2}\right) M_{[12]}^{2} M_{[34]}^{2}+\left(L_{q}^{2} R_{q^{\prime}}^{2}+R_{q}^{2} L_{q^{\prime}}^{2}\right) M_{[14]}^{2} M_{[23]}^{2}}{\left(M_{[13]}^{2}-M_{Z}^{2}\right)\left(M_{[24]}^{2}-M_{Z}^{2}\right)} .
\end{aligned}
$$

The coupling coefficients $g_{V}^{\Phi}$ are as given in table 4 . The formulae are identical to those for the case of leptonic collisions, with the replacement of the CKM matrix elements by unity for the $W^{ \pm} W^{\mp}$ fusion subprocess and the substitution of the couplings $L, R$ with the appropriate leptonic couplings $L_{e}, R_{e}$ for the $Z^{0} Z^{0}$ fusion subprocess.

For process (c), the matrix elements are given by:

$$
\begin{aligned}
\overline{|\mathcal{M}|^{2}}\left(u_{1} \bar{d}_{2} \rightarrow W_{3}^{+} \Phi_{4}\right)= & \left(g_{W^{ \pm}}^{\Phi}\right)^{2} \frac{1}{N_{C}}\left(\frac{e^{2}}{2 \sin ^{2} \theta_{W}}\right)^{2}\left(\frac{1}{2}\right)\left|V_{\mathrm{CKM}}[u d]\right|^{2} \times \\
& \times \frac{s p_{T}^{2}+2 M_{W}^{2} s}{\left(s-M_{W}^{2}\right)^{2}+M_{W}^{2} \bar{\Gamma}_{W}^{2}}, \\
\overline{|\mathcal{M}|^{2}}\left(q_{1} \bar{q}_{2} \rightarrow Z_{3} \Phi_{4}\right)= & \left(g_{Z^{0}}^{\Phi}\right)^{2} \frac{1}{N_{C}}\left(\frac{e^{2}}{4 \sin ^{2} \theta_{W} \cos ^{2} \theta_{W}}\right)^{2}\left(L_{q}^{2}+R_{q}^{2}\right) \times \\
& \times \frac{s p_{T}^{2}+2 M_{Z}^{2} s}{\left(s-M_{Z}^{2}\right)^{2}+M_{Z}^{2} \bar{\Gamma}_{Z}^{2}} .
\end{aligned}
$$


The coupling coefficients $g_{V}^{\Phi}$ are again as given in table 4 . The above formula, for the $Z^{0}$ mediated subprocess, is immediately applicable to the leptonic case with the substitution of the colour factor 1 to replace $1 / N_{C}$. The couplings $L_{q}, R_{q}$ are replaced by $L_{e}, R_{e}$ in this case.

For process (d), the matrix element for the $q \bar{q}$ initiated subprocess is given by:

$$
\begin{aligned}
\overline{|\mathcal{M}|^{2}}\left(q_{1} \bar{q}_{2} \rightarrow Q_{3} \bar{Q}_{4} \Phi_{5}\right)= & \frac{e^{2} g_{s}^{4}\left(g_{Q}^{\Phi}\right)^{2}}{2 \sin ^{2} \theta_{W} M_{W}^{2} s}\left(\frac{C_{F}}{N_{C}}\right) \mathcal{G} \times \\
& \times\left[\frac{1}{2 \mathcal{G}_{5}}-\frac{M^{2} s \mathcal{G}}{2}+M^{2}\left(\mathcal{G}_{3} p_{T 3}^{2}+\mathcal{G}_{4} p_{T 4}^{2}\right)-\mathcal{G}_{5}\left(s+M^{2}\right) p_{T 5}^{2}\right] .
\end{aligned}
$$

Here $M^{2}=M_{\Phi}^{2}$ for $A^{0}$ and $M=M_{\Phi}^{2}-4 m_{Q}^{2}$ for $h^{0}, H^{0}$. The $p_{T}$ 's are the transverse momenta of the final state particles. These can be expressed as $p_{T_{i}}^{2}=4 p_{1} \cdot p_{i} p_{2} \cdot p_{i} / s-m_{i}^{2}$ (where $i=3,4,5$ ) as the incoming particles are considered massless. The propagator factors are defined by:

$$
\begin{aligned}
\mathcal{G}_{3} & =\frac{1}{\left(M_{[35]}^{2}-m_{Q}^{2}\right)}, \\
\mathcal{G}_{4} & =\frac{1}{\left(M_{[45]}^{2}-m_{Q}^{2}\right)}, \\
\mathcal{G} & =\mathcal{G}_{3}+\mathcal{G}_{4} \\
\mathcal{G}_{5} & =\frac{\mathcal{G}_{3} \mathcal{G}_{4}}{\mathcal{G}} .
\end{aligned}
$$

For the case $Q=b$, the $g g$ initiated subprocess is evaluated with $2 \rightarrow 1,2 \rightarrow 2$ and $2 \rightarrow 3$ matrix elements. In order to avoid double counting, it is recommended that the user chooses only one of these, rather than sum over them, according to their need. For the $2 \rightarrow 1$ subprocess the matrix element is given by:

$$
\overline{|\mathcal{M}|^{2}}\left(b_{1} \bar{b}_{2} \rightarrow \Phi_{5}\right)=\frac{e^{2} M_{H}^{2} m_{b}^{2}\left(g_{b}^{\Phi}\right)^{2}}{8 \sin ^{2} \theta_{W} M_{W}^{2} N_{C}} .
$$

The $2 \rightarrow 2$ matrix element is given by:

$$
\begin{aligned}
\overline{|\mathcal{M}|^{2}}\left(g_{1} b_{2} \rightarrow b_{3} \Phi_{4}\right)= & \left(\frac{g_{s}^{2}}{2 N_{C}}\right)\left(\frac{e^{2}}{2 \sin \theta_{W}^{2}}\right)\left(\frac{m_{b}^{2}\left(g_{b}^{\Phi}\right)^{2}}{2 M_{W}^{2}}\right) \times \\
& \times\left(\frac{-u_{4}^{2}}{s t_{3}}\right)\left[1+2 \frac{m_{4}^{2}-m_{3}^{2}}{u_{4}}\left(1+\frac{m_{3}^{2}}{t_{3}}+\frac{m_{4}^{2}}{u_{4}}\right)\right] .
\end{aligned}
$$

The inconsistency in treating the initial state bottom quark as being massless and the final state bottom quark as being massive is unavoidable. The subprocess implementation being intended for high $p_{T}$ single $b$-jet events only, the ambiguity in the treatment of the bottom quark mass in the hard scattering matrix element is negligible.

We evaluated the $2 \rightarrow 3$ matrix element for $g g \rightarrow Q \bar{Q} \Phi$ using helicity amplitudes as follows. We first wrote down the amplitude functions using the corresponding Feynman rules. We then adopted the convention of ref. 16] for the heavy quark helicity eigenstates, and adopted the circular polarization for the gluons. This allowed some simplification in the computation which, together with the factorization of amplitudes, made the code very much faster than any other code which is publically available. 
For process (e), the matrix element for the $q \bar{q}$ initated subprocess is given by:

$$
\begin{aligned}
\overline{|\mathcal{M}|^{2}}\left(q_{1} \bar{q}_{2} \rightarrow \widetilde{Q}_{3} \widetilde{Q}_{4}^{*} \Phi_{5}\right)= & \left(\frac{g_{S}^{4} e^{2} g_{\widetilde{Q}}^{\Phi^{2}} M_{\widetilde{Q}}^{2}}{\sin ^{2} \theta_{W} M_{W}^{2} s}\right)\left(\frac{C_{F}}{N_{C}}\right) \times \\
& \times\left[\left|\mathcal{G}_{3}\right|^{2} p_{T 3}^{2}+\left|\mathcal{G}_{4}\right|^{2} p_{T 4}^{2}-2 \operatorname{Re}\left(\mathcal{G}_{3}^{*} \mathcal{G}_{4}\right) p_{T 3} \cdot p_{T 4}\right]
\end{aligned}
$$

The propagator factors are given by:

$$
\begin{aligned}
\mathcal{G}_{3} & =\frac{1}{\left(\left(p_{4}+p_{5}\right)^{2}-m_{3}^{2}+i m_{3} \bar{\Gamma}_{3}\right)}, \\
\mathcal{G}_{4} & =\frac{1}{\left(\left(p_{3}+p_{5}\right)^{2}-m_{4}^{2}+i m_{4} \bar{\Gamma}_{4}\right)} .
\end{aligned}
$$

The dot product of two transverse momenta is two dimensional, i.e. $p_{T 3} \cdot p_{T 4}=p_{x 3} p_{x 4}+$ $p_{y_{3}} p_{y_{4}}$.

The $g g$ initiated subprocess is again calculated using helicity amplitudes in an optimized basis. This time the two orthogonal polarisation vectors for the initial state gluons are chosen to be in the $x$ and $y$ directions, yielding a relatively compact expression:

$$
\begin{aligned}
\overline{|\mathcal{M}|^{2}}\left(g_{1} g_{2} \rightarrow \widetilde{Q}_{3} \widetilde{Q}_{4}^{*} \Phi_{5}\right)= & \left(\frac{g_{S}^{4} e^{2} g_{\widetilde{Q}}^{\Phi^{2}} M_{\widetilde{Q}}^{2} N_{C}}{4 \sin ^{2} \theta_{W} M_{W}^{2}\left(N_{C}^{2}-1\right)}\right) \times \\
& \times \sum_{i=x, y} \sum_{j=x, y}\left[\left|\mathcal{A}_{t}(i, j)\right|^{2}+\left|\mathcal{A}_{u}(i, j)\right|^{2}-\frac{1}{N_{C}^{2}} \mid \mathcal{A}_{t}(i, j)+\right. \\
& \left.+\left.\mathcal{A}_{u}(i, j)\right|^{2}\right]
\end{aligned}
$$

The colour flow components are distributed using the method of ref. [13], namely, the $\left(1 / N_{C}^{2}\right)$ term is distributed amongst the $t$-channel and $u$-channel colour flow components by their ratio. The two amplitude functions $\mathcal{A}_{t}$ and $\mathcal{A}_{u}$ are given by:

$$
\begin{aligned}
\mathcal{A}_{t}(i, j)= & 2\left(p_{i 3} p_{j_{4}} \mathcal{G}_{24} \mathcal{G}_{13}+p_{i 3} p_{{ }_{3}} \mathcal{G}_{13} \mathcal{G}_{45}+p_{i 4} p_{j_{4}} \mathcal{G}_{24} \mathcal{G}_{35}\right)- \\
& -\frac{\delta_{i j}}{\sqrt{s}}\left(p_{z 3} \mathcal{G}_{45}-p_{z 4} \mathcal{G}_{35}\right)-\frac{\delta_{i j}}{2}\left(\mathcal{G}_{35}+\mathcal{G}_{45}\right), \\
\mathcal{A}_{u}(i, j)= & 2\left(p_{i 4} p_{j_{3}} \mathcal{G}_{14} \mathcal{G}_{23}+p_{i 3} p_{j_{3}} \mathcal{G}_{23} \mathcal{G}_{45}+p_{i 4} p_{j_{4}} \mathcal{G}_{14} \mathcal{G}_{35}\right)+ \\
& +\frac{\delta_{i j}}{\sqrt{s}}\left(p_{z 3} \mathcal{G}_{45}-p_{z 4} \mathcal{G}_{35}\right)-\frac{\delta_{i j}}{2}\left(\mathcal{G}_{35}+\mathcal{G}_{45}\right) .
\end{aligned}
$$

The propagator factors are given by:

$$
\begin{aligned}
\mathcal{G}_{13} & =\frac{1}{\left(\left(p_{1}+p_{3}\right)^{2}-m_{3}^{2}\right)}, \quad \mathcal{G}_{23}=\frac{1}{\left(\left(p_{2}+p_{3}\right)^{2}-m_{3}^{2}\right)}, \\
\mathcal{G}_{14} & =\frac{1}{\left(\left(p_{1}+p_{4}\right)^{2}-m_{4}^{2}\right)}, \quad \mathcal{G}_{24}=\frac{1}{\left(\left(p_{2}+p_{4}\right)^{2}-m_{4}^{2}\right)}, \\
\mathcal{G}_{35} & =\frac{1}{\left(\left(p_{3}+p_{5}\right)^{2}-m_{4}^{2}+i m_{4} \bar{\Gamma}_{4}\right)}, \quad \mathcal{G}_{45}=\frac{1}{\left(\left(p_{4}+p_{5}\right)^{2}-m_{3}^{2}+i m_{3} \bar{\Gamma}_{3}\right)} .
\end{aligned}
$$


For process (h), the matrix element is given by:

$$
\overline{|\mathcal{M}|^{2}}\left(e_{1}^{-} e_{2}^{+} \rightarrow h_{3}^{0} A_{4}^{0}, H_{3}^{0} A_{4}^{0}\right)=e^{4} s p_{T}^{2} \cdot \frac{L_{\nu}^{2}\left(L_{e}^{2}+R_{e}^{2}\right)\left(\cos ^{2}(\beta-\alpha), \sin ^{2}(\beta-\alpha)\right)}{\left(s-M_{Z}^{2}\right)^{2}+M_{Z}^{2} \bar{\Gamma}_{Z}^{2}} .
$$

We have used the same notation as in eqs. (3.9). The coupling factor $\cos ^{2}(\beta-\alpha)$ is for $h^{0} A^{0}$ production whereas $\sin ^{2}(\beta-\alpha)$ is for $H^{0} A^{0}$ production.

\subsection{Charged Higgs production}

For hadronic collisions, the following production modes are implemented:

$$
\begin{aligned}
& g b \longrightarrow t H^{-}+\text {c.c. } ; \\
& g g \longrightarrow t \bar{b} H^{-}+\text {c.c. } ; \\
& q \bar{q} \longrightarrow t \bar{b} H^{-}+\text {c.c. } ; \\
& g g \longrightarrow \widetilde{t b^{*}} H^{-}+\text {c.c. } ; \\
& q \bar{q} \longrightarrow \widetilde{t b^{*}} H^{-}+\text {c.c. } ; \\
& q \bar{q} \longrightarrow H^{+} H^{-} ; \\
& b \bar{b} \longrightarrow W^{ \pm} H^{\mp} ; \\
& q b \longrightarrow q^{\prime} b H^{+}+\text {c.c. }
\end{aligned}
$$

Charged Higgs bosons are also produced through the decay of heavier objects including gauginos and third generation sfermions. Perhaps most notably, an important potential production mode for charged Higgs bosons is through the decay of top quarks and, as with other decay processes, this is achieved by specifying this decay mode in the input file. The decay is carried out using the three-body matrix element. This matrix element is not included in the spin correlation algorithm [17] which is the default treatment from version 6.4. If this decay is required, the matrix element code NME $=200$ should be used and spin correlations and supersymmetric three body decays should be switched off, i.e. SYSPIN $=$. FALSE . and THREEB $=$. FALSE . .

When simulating processes (3.52), (3.53) and (3.58) one should bear in mind that there is potentially a large contribution from top quark decay when this decay mode is available. In this case the user should choose to simulate either the top quark production or the relevant three-body process in order to avoid double counting. In the limit in which the bottom quark is generated by the collinear splitting $g \rightarrow b \bar{b}$, process (3.52) is described by the two body process (3.51). This contribution becomes significant if the mass of the charged Higgs boson is comparable or greater than the top quark mass. Again, the user should choose to simulate either of the two processes in order to avoid double counting.

For leptonic collisions, in addition to the top quark decay, charged Higgs bosons can be produced in a process analogous to process (3.56):

$$
e^{-} e^{+} \longrightarrow H^{+} H^{-} \text {. }
$$

The matrix element is obtained immediately from the hadronic case by changing the appropriate photon and $Z^{0}$ couplings and removing the colour factor $1 / N_{C}$. 
Process (3.51) is given by:

$$
\begin{aligned}
\overline{|\mathcal{M}|^{2}}\left(g_{1} b_{2} \rightarrow t_{3} H_{4}^{-}\right)= & \left(\frac{g_{s}^{2}}{2 N_{C}}\right)\left(\frac{e^{2}}{2 \sin \theta_{W}^{2}}\right)\left|V_{\mathrm{CKM}}[t b]\right|^{2}\left(\frac{m_{b}^{2} \tan ^{2} \beta+m_{t}^{2} \cot ^{2} \beta}{2 M_{W}^{2}}\right) \times \\
& \times\left(\frac{-u_{4}^{2}}{s t_{3}}\right)\left[1+2 \frac{m_{4}^{2}-m_{3}^{2}}{u_{4}}\left(1+\frac{m_{3}^{2}}{t_{3}}+\frac{m_{4}^{2}}{u_{4}}\right)\right] .
\end{aligned}
$$

Process (3.52) is evaluated using helicity amplitudes in an optimized basis, similarly to the evaluation of the neutral Higgs boson production process $g g \rightarrow Q \bar{Q} \Phi$ in section 3.5.

Process (3.53) is evaluated using a more general formula for $q_{1} \bar{q}_{2} \rightarrow Q_{3} \bar{Q}_{4} \Phi$ which is given by:

$$
\begin{aligned}
\overline{|\mathcal{M}|^{2}}\left(q_{1} \bar{q}_{2} \rightarrow Q_{3}\right. & \left.\bar{Q}_{4} \Phi\right)= \\
=\left(\frac{g_{s}^{4} C_{F}}{2 N_{C}}\right) \frac{2 \phi_{H}^{2}}{s} & {\left[\left(p_{T_{3}}^{2}+p_{T_{4}}^{2}-p_{T_{5}}^{2}\right)+4 p_{0} \cdot p_{3} p_{0} \cdot p_{4}|\mathcal{G}|^{2}+\right.} \\
& +2\left(p_{3} \cdot p_{4}-\lambda m_{3} m_{4}\right)\left[-s|\mathcal{G}|^{2}+2 \operatorname{Re}\left(\mathcal{G G}_{3}^{*} p_{T_{4}}^{2}+\mathcal{G G}_{4}^{*} p_{T_{3}}^{2}-\mathcal{G}_{3} \mathcal{G}_{4}^{*} p_{T_{5}}^{2}\right)\right]- \\
& -4 \operatorname{Re}\left[\mathcal{G G}_{3}^{*} p_{T_{4}}^{2}+\mathcal{G G}_{4}^{*} p_{T_{3}}^{2}+\mathcal{G}\left(\mathcal{G}_{3}^{*} p_{0} \cdot p_{4}+\mathcal{G}_{4}^{*} p_{0} \cdot p_{3}\right) \frac{p_{T_{3}}^{2}+p_{T_{4}}^{2}-p_{T_{5}}^{2}}{2}\right]- \\
& \left.-4 \operatorname{Im}\left[\mathcal{G G}_{3}^{*} p_{z_{4}}-\mathcal{G G}_{4}^{*} p_{z_{3}}\right] \lambda^{\prime} \sqrt{s}\left(p_{x_{4}} p_{y_{3}}-p_{x_{3}} p_{y_{4}}\right)\right]
\end{aligned}
$$

with $p_{0}=p_{1}+p_{2}$. Denoting the $Q_{3} \bar{Q}_{4} \Phi$ vertex as $\left(g_{\mathrm{S}}+g_{\mathrm{P}} \gamma_{5}\right)$, the various coupling coefficients appearing above are defined by:

$$
\begin{aligned}
\phi_{H}^{2} & =g_{\mathrm{S}}^{2}+g_{\mathrm{P}}^{2}, \\
\lambda & =\frac{g_{\mathrm{S}}^{2}-g_{\mathrm{P}}^{2}}{\phi_{H}^{2}}, \\
\lambda^{\prime} & =\frac{2 g_{\mathrm{S}} g_{\mathrm{P}}}{\phi_{H}^{2}} .
\end{aligned}
$$

Process (3.54) is evaluated using helicity amplitudes. The expressions are the same as eqs. (3.47)-(3.49) but the coupling coefficient needs to be changed. Let us consider the vertex involving an incoming $H^{+}$and outgoing $\widetilde{t}_{i} \widetilde{b}_{j}^{*}$. We have the following replacement:

$$
\begin{aligned}
\frac{g_{\widetilde{Q}} M_{\widetilde{Q}}^{2}}{M_{W}^{2}} \longrightarrow \frac{1}{\sqrt{2}}[ & \left(\sin 2 \beta-\frac{m_{b}^{2} \tan \beta+m_{t}^{2} \cot \beta}{M_{W}^{2}}\right) Q_{L i}^{t} Q_{L j}^{b}- \\
& -\frac{m_{t} m_{b}}{M_{W}^{2}}(\tan \beta+\cot \beta) Q_{R i}^{t} Q_{R j}^{b}-\frac{m_{b}}{M_{W}^{2}}\left(-\mu+A_{b} \tan \beta\right) Q_{L i}^{t} Q_{R j}^{b}- \\
& \left.-\frac{m_{t}}{M_{W}^{2}}\left(-\mu+A_{t} \cot \beta\right) Q_{R i}^{t} Q_{L j}^{b}\right]
\end{aligned}
$$

Process 3.56$)$ is given by:

$$
\begin{aligned}
\overline{|\mathcal{M}|^{2}}= & \frac{e^{4} s p_{T}^{2}}{N_{C}} \times \\
& \times\left[\left|\frac{Q_{q} Q_{H^{+}}}{s}+\frac{L_{q} L_{H^{+}}}{s-M_{Z}^{2}+i M_{Z} \bar{\Gamma}_{Z}}\right|^{2}+\left|\frac{Q_{q} Q_{H^{+}}}{s}+\frac{R_{q} L_{H^{+}}}{s-M_{Z}^{2}+i M_{Z} \bar{\Gamma}_{Z}}\right|^{2}\right] .
\end{aligned}
$$


We have used the same notation as in eq. (3.9) $\cdot Q_{H^{+}}=+1$, and $L_{H^{+}}$is given by eq. (3.6) with $I_{f}^{3}=+1 / 2$. For the leptonic case the subscripts $q$ should be replaced with $e$, and the colour factor $1 / N_{C}$ should be replaced by 1 .

The matrix element for process (3.57) is as given in [18].

Process (3.58) is given in [19]. We use a more compact expression which sets the kinematic bottom quark mass to zero. For the process $b_{1} U_{2} \rightarrow b_{3} D_{4} H_{5}^{+}$we have:

$$
\begin{aligned}
\overline{|\mathcal{M}|^{2}}\left(b_{1} U_{2} \rightarrow b_{3} D_{4} H_{5}^{+}\right)= & \frac{1}{2}\left(\frac{e^{2}}{2 x_{W}}\right)^{3}\left|V_{\mathrm{CKM}}[U D]\right|^{2} \frac{1}{\left(M_{[24]}^{2}-M_{W}^{2}\right)^{2}} \times \\
& \left.\times\left[\left|V_{\mathrm{CKM}}[t b]\right|^{2} \overline{\left|A_{t}\right|^{2}}+\overline{\left|A_{\Phi}\right|^{2}}+\operatorname{Re} V_{\mathrm{CKM}}[t b] \cdot \overline{2 A_{\Phi}^{*} A_{t}}\right)\right] ; \\
\overline{\left|A_{t}\right|^{2}}= & 2 M_{[12]}^{2}\left|\mathcal{G}_{t}\right|^{2}\left[\left(\frac{m_{b} t_{\beta}}{m_{W}}\right)^{2} \frac{[3545]}{2}+\left(\frac{m_{t} t_{\beta}^{-1}}{m_{W}}\right)^{2} m_{t}^{2} M_{[34]}^{2}\right] ; \\
\overline{\left|A_{\Phi}\right|^{2}}= & \left(\mathcal{G}_{h, H}^{2}+\mathcal{G}_{A}^{2}\right)\left(-M_{[13]}^{2}\right) \frac{[2545]}{2} ; \\
\operatorname{Re}\left(\overline{2 A_{\Phi}^{*} A_{t}}\right)= & \frac{m_{b} t_{\beta}}{M_{W}}\left(\mathcal{G}_{h, H}+\mathcal{G}_{A}\right) \times \\
& \times\left[2 \operatorname{Re}\left(\mathcal{G}_{t}\right)\left(M_{[45]}^{2}-M_{H^{ \pm}}^{2}\right)\left(M_{[12]}^{2}\right)\left(-M_{[13]}^{2}\right)-\right. \\
& \left.-\operatorname{Re}\left(\mathcal{G}_{t}\right) M_{[45]}^{2}[1243]+\operatorname{Im}\left(\mathcal{G}_{t}\right) M_{[45]}^{2} \cdot 4 \operatorname{det}(1234)\right] .
\end{aligned}
$$

Here we have defined $[a b c d]=4\left(p_{a} \cdot p_{b} p_{c} \cdot p_{d}+p_{a} \cdot p_{d} p_{b} \cdot p_{c}-p_{a} \cdot p_{c} p_{b} \cdot p_{d}\right)$ and $\operatorname{det}(a b c d)=$ $\operatorname{det}\left(p_{a}^{\mu}, p_{b}^{\nu}, p_{c}^{\rho}, p_{d}^{\sigma}\right)=\sum_{\mu \nu \rho \sigma} \epsilon_{\mu \nu \rho \sigma} p_{a}^{\mu} p_{b}^{\nu} p_{c}^{\rho} p_{d}^{\sigma}$ where $\epsilon_{0123}=+1$.

The propagators are defined by:

$$
\begin{aligned}
\mathcal{G}_{t} & =\frac{1}{M_{[35]}^{2}-m_{t}^{2}+i m_{t} \bar{\Gamma}_{t}}=\frac{M_{[35]}^{2}-m_{t}^{2}-i m_{t} \bar{\Gamma}_{t}}{\left(M_{[35]}^{2}-m_{t}^{2}\right)^{2}+m_{t}^{2} \bar{\Gamma}_{t}^{2}} ; \\
\mathcal{G}_{h, H} & =\frac{m_{b} \sin \alpha \cos (\beta-\alpha) / M_{W} \cos \beta}{M_{[13]}^{2}-M_{h}^{2}}+\frac{m_{b} \cos \alpha \sin (\beta-\alpha) / M_{W} \cos \beta}{M_{[13]}^{2}-M_{H}^{2}} \\
\mathcal{G}_{A} & =\frac{m_{b} \tan \beta / M_{W}}{M_{[13]}^{2}-M_{A}^{2}} .
\end{aligned}
$$

Processes with antiquarks in the initial state can be obtained by exchanging the subscripts $(1 \leftrightarrow 3)$ for the subprocess $\bar{b} U \rightarrow \bar{b} D H^{+},(2 \leftrightarrow 4)$ for the subprocess $b \bar{D} \rightarrow b \bar{U} H^{+}$, and both for the subprocess $\bar{b} \bar{D} \rightarrow \bar{b} \bar{U} H^{+}$. Note that the definitions of the invariant masses squared are such that these imply momentum substitutions of the form $\left(p_{1} \leftrightarrow-p_{3}\right)$.

\subsection{R-parity violating processes}

The cross sections for the implemented R-parity violating hadron-hadron processes are presented in ref. [20]. In hadron-hadron collisions only those $2 \rightarrow 2$ processes that can occur via a resonant $s$-channel sparticle exchange are included, i.e. processes that can only occur via a $t$-channel diagram or for which the $s$-channel resonance is not kinematically accessible are neglected. 
In $e^{+} e^{-}$collisions we include all the possible $2 \rightarrow 2$ single sparticle production mechanisms, even if there is no $s$-channel resonance, and a limited set of $2 \rightarrow 2$ processes where the final state contains two SM particles. As with the hadron-hadron processes, there are four types of process: single gaugino production; single slepton production in association with a gauge boson; single slepton production in association with a Higgs boson; SM particle production.

\subsubsection{Single gaugino production}

In $e^{+} e^{-}$collisions, there are two possible production mechanisms for a gaugino and a lepton, either $e^{+} e^{-} \rightarrow \widetilde{\chi}^{0} \nu$ or $e^{+} e^{-} \rightarrow \tilde{\chi}^{+} \ell^{-}$. The matrix elements for these processes have been calculated previously in refs. [21, 22, 23. We have recalculated these matrix elements and the results are in agreement with [23]. We also agree with refs. [21, 22, which do not include sfermion mixing, if we set our mixing to zero. All matrix elements are averaged over the spins of the incoming leptons as before.

$$
\begin{aligned}
\overline{|\mathcal{M}|^{2}}\left(e_{i}^{-} e_{i}^{+} \rightarrow \nu_{j} \widetilde{\chi}_{l}^{0}\right)= & \lambda_{i j i}^{2}\left[\left|a\left(\widetilde{\nu}_{j}^{*}\right)\right|^{2} s R\left(\widetilde{\nu}_{j}\right)\left(s-M_{\widetilde{\chi}_{l}^{0}}^{2}\right)+\frac{1}{\left(u-M_{\ell_{i L}}^{2}\right)^{2}}\left[\left|a\left(\widetilde{\ell}_{i 1}^{*}\right)\right|^{2}+\left|b\left(\widetilde{\ell}_{i 1}^{*}\right)\right|^{2}\right] u\left(u-M_{\widetilde{\chi}_{l}^{0}}^{2}\right)+\right. \\
+ & \frac{1}{\left(t-M_{\ell_{i R}}^{2}\right)^{2}}\left[\left|a\left(\widetilde{\ell}_{i 2}\right)\right|^{2}+\left|b\left(\widetilde{\ell}_{i 2}\right)\right|^{2}\right] t\left(t-M_{\widetilde{\chi}_{l}^{0}}^{2}\right)+ \\
& +\frac{2}{\left(u-M_{\ell_{i L}}^{2}\right)} a\left(\widetilde{\nu}_{j}^{*}\right) a\left(\widetilde{\ell}_{i 1}^{*}\right) R\left(\widetilde{\nu}_{j}\right)\left(s-m_{\widetilde{\nu}_{j}}^{2}\right) s u+ \\
+ & \frac{2}{\left(u-M_{\ell_{i L}}^{2}\right)\left(t-M_{\ell_{i R}}^{2}\right)} a\left(\widetilde{\ell}_{i 1}^{*}\right) a\left(\widetilde{\ell}_{i 2}\right) u t+ \\
\hline \overline{\left.\mathcal{M}\right|^{2}}\left(e_{i}^{-} e_{i}^{+}\right. & \left.\rightarrow \frac{2}{\left(t-M_{\ell_{i R}}^{2}\right)} a\left(\widetilde{\nu}_{j}^{*}\right) a\left(\widetilde{\ell}_{l}^{-}\right)=R\left(\widetilde{\nu}_{j}\right)\left(s-m_{\widetilde{\nu}_{j}}^{2}\right) s t\right], \\
=\frac{g^{2} \lambda_{i j i}^{2}}{4} & {\left[R\left(\widetilde{\nu}_{j}\right) s\left[\left(\left|a\left(\widetilde{\nu}_{j}^{*}\right)\right|^{2}+\left|b\left(\widetilde{\nu}_{j}^{*}\right)\right|^{2}\right)\left(s-m_{\ell_{j}}^{2}-M_{\widetilde{\chi}_{l}^{-}}^{2}\right)-4 a\left(\widetilde{\nu}_{j}^{*}\right) b\left(\widetilde{\nu}_{j}^{*}\right) m_{\ell_{j}} M_{\widetilde{\chi}_{l}^{-}}\right]+\right.} \\
& +\frac{1}{\left(u-M_{\widetilde{\nu}_{i}}^{2}\right)^{2}}\left(M_{\widetilde{\chi}_{l}^{-}}^{2}-u\right)\left(m_{\ell_{j}}^{2}-u\right)\left[\left|a\left(\widetilde{\nu}_{i}^{*}\right)\right|^{2}+\left|b\left(\widetilde{\nu}_{i}^{*}\right)\right|^{2}\right]- \\
& \left.-\frac{2 s}{\left(u-M_{\widetilde{\nu}_{i}}^{2}\right)}\left(s-M_{\widetilde{\nu}_{j}}^{2}\right) R\left(\widetilde{\nu}_{j}\right) a\left(\widetilde{\nu}_{i}^{*}\right)\left[a\left(\widetilde{\nu}_{j}^{*}\right) u+m_{\ell_{j}} M_{\widetilde{\chi}_{l}^{-}} b\left(\widetilde{\nu}_{j}^{*}\right)\right]\right] .
\end{aligned}
$$

The couplings $a, b$ of the sleptons to the gauginos are given in ref. [20], and

$$
R(\tilde{a})=\frac{1}{\left(s-M_{\tilde{a}}^{2}\right)^{2}+\bar{\Gamma}_{\tilde{a}}^{2} M_{\tilde{a}}^{2}} .
$$




\subsubsection{Slepton production in association with a gauge boson}

There are a number of production processes for a slepton in association with a gauge boson. We have confirmed the cross section for these processes, which were first calculated in ref. [24]. The sneutrino resonance is not usually accessible for these processes as the charged slepton-sneutrino mass difference is less than the $W^{ \pm}$mass. However, the resonance can be accessible for the third generation due to left-right mixing of the staus.

The matrix elements for these processes are given below, including left-right sfermion mixing:

$$
\begin{aligned}
& \overline{|\mathcal{M}|^{2}}\left(e_{i}^{-} e_{i}^{+} \rightarrow \gamma \widetilde{\nu}_{j}\right)= \frac{e^{2} \lambda_{i j i}^{2}}{2}\left[\frac{u}{t}+\frac{t}{u}+\frac{2}{u t}\left(M_{\widetilde{\nu}_{j}}^{2}-u\right)\left(M_{\widetilde{\nu}_{j}}^{2}-t\right)\right], \\
& \overline{|\mathcal{M}|^{2}}\left(e_{i}^{-} e_{i}^{+} \rightarrow W^{+} \widetilde{\ell}_{j \alpha}\right)= \\
&=\frac{g^{2} \lambda_{i j i}^{2}\left|L_{1 \alpha}^{j}\right|^{2}}{2 M_{W}^{2}}\left[s^{2} p_{\mathrm{cm}}^{2} R\left(\widetilde{\nu}_{j}\right)+\frac{1}{4 u^{2}}\left[2 M_{W}^{2}\left(u t-M_{W}^{2} M_{\widetilde{\ell}_{j \alpha}}^{2}\right)+u^{2} s\right]-\right. \\
&\left.-\frac{s}{2 u} R\left(\widetilde{\nu}_{j}\right)\left(s-M_{\widetilde{\nu}_{j}}^{2}\right)\left[M_{W}^{2}\left(2 M_{\widetilde{\ell}_{j \alpha}}^{2}-u\right)+u\left(s-M_{\widetilde{\ell}_{j \alpha}}^{2}\right)\right]\right], \\
& \overline{|\mathcal{M}|^{2}}\left(e_{i}^{-} e_{i}^{+} \rightarrow Z^{0} \widetilde{\nu}_{j}\right)= \\
&=\frac{g^{2} \lambda_{i j i}^{2}}{\cos ^{2} \theta_{W} M_{Z}^{2}}[\left|Z_{\nu_{j}}^{11}\right|^{2} s^{2} p_{\mathrm{cm}}^{2} R\left(\widetilde{\nu}_{j}\right)+\frac{Z_{e_{R}}^{2}}{t^{2}}\left[2 M_{Z}^{2}\left(u t-M_{\widetilde{\nu}_{j}}^{2} M_{Z}^{2}\right)+s t^{2}\right]+ \\
&+\frac{Z_{e_{L}}^{2}}{u^{2}}\left[2 M_{Z}^{2}\left(u t-M_{\widetilde{\nu}_{j}}^{2} M_{Z}^{2}\right)+s u^{2}\right]+ \\
&+\frac{2 Z_{e_{L}} Z_{e_{R}}}{u t}\left[2 M_{Z}^{2}\left(M_{\widetilde{\nu}_{j}}^{2}-t\right)\left(M_{\widetilde{\nu}_{j}}^{2}-u\right)-s u t\right]- \\
&-\frac{Z_{\widetilde{\nu}_{j}}^{11} Z_{e_{R}}}{t} s R\left(\widetilde{\nu}_{j}\right)\left(s-M_{\widetilde{\nu}_{j}}^{2}\right)\left[M_{Z}^{2}\left(2 M_{\widetilde{\nu}_{j}}^{2}-t\right)+t\left(s-M_{\widetilde{\nu}_{j}}^{2}\right)\right]+ \\
&\left.+\frac{Z_{\widetilde{\nu}_{j}}^{11} Z_{e_{L}}}{u} s R\left(\widetilde{\nu}_{j}\right)\left(s-M_{\widetilde{\nu}_{j}}^{2}\right)\left[M_{Z}^{2}\left(2 M_{\widetilde{\nu}_{j}}^{2}-u\right)+u\left(s-M_{\widetilde{\nu}_{j}}^{2}\right)\right]\right],
\end{aligned}
$$

where $p_{\mathrm{cm}}$ is the momentum of the final-state particles in the centre-of-mass frame. As we neglect the electron mass, the cross section for $e_{i}^{-} e_{i}^{+} \rightarrow \gamma \widetilde{\nu}_{j}$ is divergent as $u, t \rightarrow 0$, i.e. in the limit that the photon is collinear with the incoming lepton beams, and therefore a cut on the transverse momenta of the outgoing particles is used to regularize the divergence for this process. The couplings of the $Z^{0}$ to the leptons and sleptons are given in ref. 20].

\subsubsection{Slepton production in association with a Higgs boson}

There are a number of processes in which a Higgs boson can be produced in association with a slepton. The cross sections for these processes tend to be small due to the small lepton masses and the inaccessibility of the sneutrino resonance as the charged slepton-sneutrino mass difference is smaller than the charged Higgs mass. 
The matrix elements are given below

$$
\begin{aligned}
\overline{|\mathcal{M}|^{2}}\left(e_{i}^{-} e_{i}^{+} \rightarrow \widetilde{\ell}_{j \alpha}^{*} H^{-}\right)=\frac{g^{2} \lambda_{i j i}^{2}}{4}\left[\left|H_{\widetilde{\nu} \widetilde{\ell}_{j \alpha}}^{c}\right|^{2} s R\left(\widetilde{\nu}_{j}\right)+\frac{4\left|L_{1 \alpha}^{2 j-1}\right|^{2}\left|E_{j}^{c}\right|^{2}}{u^{2}}\left(u t-M_{\widetilde{\ell}_{j \beta}}^{2} M_{H^{-}}^{2}\right)\right], \\
\overline{|\mathcal{M}|^{2}}\left(e_{i}^{-} e_{i}^{+} \rightarrow \widetilde{\nu}_{j}^{*} H_{l}^{0}\right)=\frac{g^{2} \lambda_{i j i}^{2}}{4}\left[\left|H_{\widetilde{\nu}_{j} \widetilde{\nu}_{j}}^{l}\right|^{2} s R\left(\widetilde{\nu}_{j}\right)+\frac{\left|E_{i}^{l}\right|^{2}}{u^{2}}\left(u t-M_{\widetilde{\nu}_{j}}^{2} M_{H_{l}^{0}}^{2}\right)+\right. \\
\left.+\frac{\left|E_{i}^{l}\right|^{2}}{t^{2}}\left(u t-M_{\widetilde{\nu}_{j}}^{2} M_{H_{l}^{0}}^{2}\right)\right] .
\end{aligned}
$$

The couplings $H$ of the Higgs bosons to the sleptons are given in ref. [20] and the couplings $E_{i}^{l}$ of the leptons to the Higgs bosons can be obtained from the couplings given in ref. [20] for the down-type quarks by replacing the quark mass with the lepton mass.

\subsubsection{SM particle production by sparticle exchange}

As with the hadron-hadron processes described in ref. [20] we have only included $2 \rightarrow$ 2 processes where two SM particles can be produced by a resonant $s$-channel exchange. Where this is possible we have included any $t$-channel sparticle exchanges, and the relevant interference terms. We have also included the relevant SM diagrams and the interference between the SM and the R-parity violating diagrams. We have generalized the results of ref. [25], giving

$$
\begin{aligned}
\overline{|\mathcal{M}|^{2}}\left(e_{i}^{-} e_{i}^{+} \rightarrow f_{k} \bar{f}_{l}\right)=\frac{e^{4} s^{2} C_{\mathrm{fac}}}{4}\left\{(1+\cos \theta)^{2}[\right. & \left|f_{L R}^{s}\right|^{2}+\left|f_{R L}^{s}\right|^{2}+\left|f_{L R}^{t}\right|^{2}+\left|f_{R L}^{t}\right|^{2}+ \\
& \left.+2 \operatorname{Re}\left(f_{L R}^{s *} f_{L R}^{t}\right)+2 \operatorname{Re}\left(f_{R L}^{s *} f_{R L}^{t}\right)\right]+ \\
& +(1-\cos -\theta)^{2}\left[\left|f_{L L}^{s}\right|^{2}+\left|f_{R R}^{s}\right|^{2}\right]+ \\
& \left.+4\left[\left|f_{L L}^{t}\right|^{2}+\left|f_{R R}^{t}\right|^{2}\right]\right\},
\end{aligned}
$$

where the coefficients are given by

$$
\begin{aligned}
& f_{L L}^{s}=\delta_{l k}\left\{\frac{1}{s}+\frac{L_{e} R_{e}}{s-M_{Z}^{2}+i \bar{\Gamma}_{Z} M_{Z}}\right\}+\sum_{j=1,3} \frac{\lambda_{j i k} \lambda_{j i l}}{2 e^{2}\left(t-M_{\widetilde{\nu}_{j}}^{2}\right)} \\
& f_{L R}^{s}=\delta_{l k}\left\{\frac{1}{s}+\frac{L_{e}^{2}}{s-M_{Z}^{2}+i \bar{\Gamma}_{Z} M_{Z}}\right\}, \quad f_{R L}^{s}=\delta_{l k}\left\{\frac{1}{s}+\frac{R_{e}^{2}}{s-M_{Z}^{2}+i \bar{\Gamma}_{Z} M_{Z}}\right\} \\
& f_{R R}^{s}=\delta_{l k}\left\{\frac{1}{s}+\frac{L_{e} R_{e}}{s-M_{Z}^{2}+i \bar{\Gamma}_{Z} M_{Z}}\right\}+\sum_{j=1,3} \frac{\lambda_{j k i} \lambda_{j l i}}{2 e^{2}\left(t-M_{\widetilde{\nu}_{j}}^{2}\right)}, \\
& f_{L L}^{t}=\delta_{i l} \delta_{l k}\left\{\frac{1}{t}+\frac{L_{e} R_{e}}{t-M_{Z}^{2}}\right\}+\sum_{j=1,3} \frac{\lambda_{j i i} \lambda_{j k l}}{2 e^{2}\left(s-M_{\widetilde{\nu}_{j}}^{2}+i \bar{\Gamma}_{\widetilde{\nu}_{j}} M_{\widetilde{\nu}_{j}}\right)} \\
& \left.f_{R R}^{t}=\delta_{i l} \delta_{l k}\left\{\frac{1}{t}+\frac{L_{e}{ }^{2}}{t-M_{Z}^{2}}\right\}, \quad f_{R L}^{t}=\delta_{i l} \delta_{l k}\left\{\frac{1}{t}+\frac{R_{e}}{t-M_{l k}^{2}}\right\} \frac{1}{t}+\frac{L_{e} R_{e}}{t-M_{Z}^{2}}\right\}+\sum_{j=1,3} \frac{\lambda_{j i i} \lambda_{j l k}}{2 e^{2}\left(s-M_{\widetilde{\nu}_{j}}^{2}+i \bar{\Gamma}_{\widetilde{\nu}_{j}} M_{\widetilde{\nu}_{j}}\right)}
\end{aligned}
$$


for $e_{i}^{-} e_{i}^{+} \rightarrow e_{k}^{-} e_{l}^{+}$and by

$$
\begin{aligned}
& f_{L L}^{s}=\delta_{l k}\left\{-\frac{e_{d}}{s}+\frac{L_{e} R_{d}}{s-M_{Z}^{2}+i \bar{\Gamma}_{Z} M_{Z}}\right\}+\sum_{j=1,3} \sum_{\alpha=1,2} \frac{\lambda_{i j k}^{\prime} \lambda_{i j l}^{\prime}}{2 e^{2}\left(t-M_{\widetilde{u}_{j \alpha}}^{2}\right)}, \\
& f_{L R}^{s}=\delta_{l k}\left\{-\frac{e_{d}}{s}+\frac{L_{e} L_{d}}{s-M_{Z}^{2}+i \bar{\Gamma}_{Z} M_{Z}}\right\}, \quad f_{R L}^{s}=\delta_{l k}\left\{-\frac{e_{d}}{s}+\frac{R_{e} R_{d}}{s-M_{Z}^{2}+i \bar{\Gamma}_{Z} M_{Z}}\right\}, \\
& f_{R R}^{s}=\delta_{l k}\left\{-\frac{e_{d}}{s}+\frac{R_{e} L_{d}}{s-M_{Z}^{2}+i \bar{\Gamma}_{Z} M_{Z}}\right\}, \quad f_{L L}^{t}=\sum_{j=1,3} \frac{\lambda_{j i i} \lambda_{j k l}^{\prime}}{2 e^{2}\left(s-M_{\widetilde{\nu}_{j}}^{2}+i \bar{\Gamma}_{\widetilde{\nu}_{j}} M_{\widetilde{\nu}_{j}}\right)}, \\
& f_{L R}^{t}=0, \quad f_{R L}^{t}=0, \quad f_{R R}^{t}=\sum_{j=1,3} \frac{\lambda_{j i i} \lambda_{j l k}^{\prime}}{2 e^{2}\left(s-M_{\widetilde{\nu}_{j}}^{2}+i \bar{\Gamma}_{\widetilde{\nu}_{j}} M_{\widetilde{\nu}_{j}}\right)},
\end{aligned}
$$

for $e_{i}^{-} e_{i}^{+} \rightarrow d_{k} \bar{d}_{l}$. The colour factor $C_{\mathrm{fac}}$ is 1 for charged lepton production and $N_{C}$ for quark production. The left/right charges of the fermions are given in section 3.1. This formula corrects the sign of the interference term given in [25], which is necessary both to reproduce their numerical results and to obtain the correct SM Bhabha cross section formula in the limit that the R-parity violating couplings are zero.

\subsection{Decay matrix elements}

The decay matrix elements are implemented for both R-parity conserving and R-parity violating three-body decays of the gluino and the gauginos. The R-parity conserving decays are performed using the helicity amplitude expressions given in ref. [17]. The R-parity violating formulae are as given in ref. [20].

Spin correlations in the distribution of cascade decay products are implemented in all decay processes, as well as the R-parity conserving $2 \rightarrow 2$ SUSY production processes. The implementation is as described in ref. [177].

\section{Conclusions}

The Monte Carlo event generator HERWIG has been extended to include the simulation of supersymmetry. We have summarised its salient features. We have defined the parameter conventions and listed the matrix elements for all original calculations.

\section{Acknowledgments}

We thank the co-authors of HERWIG for fruitful collaboration, and the members of the Cavendish ATLAS-HERWIG working group for stimulating discussions and for testing part of our code. P.R. would like to thank H. Dreiner for many useful discussions on the implementation of R-parity violating processes. S.M. thanks M.L. Mangano, H. Dreiner and M. Krämer for many useful discussions and the latter in particular for making available an independent implementation of the leading order matrix elements for gaugino production. 


\section{A. Process codes}

We summarize the process codes IPROC for MSSM hard scattering processes implemented in HERWIG.

\begin{tabular}{|c|c|}
\hline IPROC & Process \\
\hline $700-99$ & R-parity conserving SUSY processes \\
\hline 700 & $\ell^{+} \ell^{-} \rightarrow 2$-sparticle processes (sum of $710-760$ ) \\
\hline 710 & $\ell^{+} \ell^{-} \rightarrow$ neutralino pairs (all neutralinos) \\
\hline $706+4 \mathrm{IN} 1+\mathrm{IN} 2$ & $\ell^{+} \ell^{-} \rightarrow \widetilde{\chi}_{\mathrm{IN} 1}^{0} \tilde{\chi}_{\mathrm{IN} 2}^{0}(\mathrm{IN} 1,2=$ neutralino mass eigenstate $)$ \\
\hline 730 & $\ell^{+} \ell^{-} \rightarrow$ chargino pairs (all charginos) \\
\hline $728+2 \mathrm{IC} 1+\mathrm{IC} 2$ & $\ell^{+} \ell^{-} \rightarrow \tilde{\chi}_{\mathrm{IC} 1}^{+} \tilde{\chi}_{\mathrm{IC} 2}^{-}(\mathrm{IC} 1,2=$ chargino mass eigenstate $)$ \\
\hline 740 & $\ell^{+} \ell^{-} \rightarrow$ slepton pairs (all flavours) \\
\hline $736+5 \mathrm{IL}$ & $\ell^{+} \ell^{-} \rightarrow \widetilde{\ell}_{L, R} \widetilde{\ell}_{L, R}^{*}(\mathrm{IL}=1,2,3$ for $\tilde{\ell}=\tilde{e}, \tilde{\mu}, \tilde{\tau})$ \\
\hline $737+5 \mathrm{IL}$ & $\ell^{+} \ell^{-} \rightarrow \widetilde{\ell}_{L} \widetilde{\ell}_{L}^{*}$ (IL as above) \\
\hline $738+5 \mathrm{IL}$ & $\ell^{+} \ell^{-} \rightarrow \widetilde{\ell}_{L} \widetilde{\ell}_{R}^{*}$ (IL as above) \\
\hline $739+5 \mathrm{IL}$ & $\ell^{+} \ell^{-} \rightarrow \widetilde{\ell}_{R} \widetilde{\ell}_{R}^{*}$ (IL as above) \\
\hline $740+5 \mathrm{IL}$ & $\ell^{+} \ell^{-} \rightarrow \widetilde{\nu}_{L} \widetilde{\nu}_{L}^{*}\left(\mathrm{IL}=1,2,3\right.$ for $\left.\widetilde{\nu}_{e}, \widetilde{\nu}_{\mu}, \widetilde{\nu}_{\tau}\right)$ \\
\hline 760 & $\ell^{+} \ell^{-} \rightarrow$ squark pairs (all flavours) \\
\hline $757+4 \mathrm{IQ}$ & $\ell^{+} \ell^{-} \rightarrow \widetilde{q}_{L, R} \widetilde{q}_{L, R}^{*}(\mathrm{IQ}=1, \ldots, 6$ for $\widetilde{q}=\tilde{d}, \ldots, \tilde{t})$ \\
\hline $758+4 \mathrm{IQ}$ & $\ell^{+} \ell^{-} \rightarrow \widetilde{q}_{L} \widetilde{q}_{L}^{*}($ IQ as above $)$ \\
\hline $759+4 \mathrm{IQ}$ & $\ell^{+} \ell^{-} \rightarrow \widetilde{q}_{L} \widetilde{q}_{R}^{*}$ (IQ as above) \\
\hline $760+4 \mathrm{IQ}$ & $\ell^{+} \ell^{-} \rightarrow \widetilde{q}_{R} \widetilde{q}_{R}^{*}$ (IQ as above) \\
\hline $800-99$ & R-parity violating SUSY processes \\
\hline 800 & Single sparticle production, sum of $810-840$ \\
\hline 810 & $\ell^{+} \ell^{-} \rightarrow \tilde{\chi}^{0} \nu_{i}$, (all neutralinos) \\
\hline $810+\mathrm{IN}$ & $\ell^{+} \ell^{-} \rightarrow \tilde{\chi}_{\mathrm{IN}}^{0} \nu_{i},(\mathrm{IN}=$ neutralino mass state $)$ \\
\hline 820 & $\ell^{+} \ell^{-} \rightarrow \tilde{\chi}^{-} e_{i}^{+}$(all charginos) \\
\hline $820+\mathrm{IC}$ & $\ell^{+} \ell^{-} \rightarrow \tilde{\chi}_{\mathrm{IC}}^{-} e_{i}^{+},(\mathrm{IC}=$ chargino mass state $)$ \\
\hline 830 & $\ell^{+} \ell^{-} \rightarrow \widetilde{\nu}_{i} Z^{0}$ and $\ell^{+} \ell^{-} \rightarrow \widetilde{\ell}_{i}^{+} W^{-}$ \\
\hline 840 & $\ell^{+} \ell^{-} \rightarrow \widetilde{\nu}_{i} h^{0} / H^{0} / A^{0}$ and $\ell^{+} \ell^{-} \rightarrow \widetilde{\ell}_{i}^{+} H^{-}$ \\
\hline 850 & $\ell^{+} \ell^{-} \rightarrow \widetilde{\nu}_{i} \gamma$ \\
\hline 860 & Sum of 870 and 880 \\
\hline 870 & $\ell^{+} \ell^{-} \rightarrow \ell^{+} \ell^{-}$, via LLE only \\
\hline $867+3 \mathrm{IL} 1+\mathrm{IL} 2$ & $\ell^{+} \ell^{-} \rightarrow \ell_{\mathrm{IL} 1}^{+} \ell_{\mathrm{IL} 2}^{-}(\mathrm{IL} 1,2=1,2,3$ for $e, \mu, \tau)$ \\
\hline 880 & $\ell^{+} \ell^{-} \rightarrow \bar{d} d$, via $\mathrm{LLE}$ and LQD \\
\hline $877+3 \mathrm{IQ} 1+\mathrm{IQ} 2$ & $\ell^{+} \ell^{-} \rightarrow d_{\mathrm{IL} 1} \bar{d}_{\mathrm{IL} 2}(\mathrm{IQ} 1,2=1,2,3$ for $d, s, b)$ \\
\hline 910 & $\ell^{+} \ell^{-} \rightarrow \nu_{e} \bar{\nu}_{e} h^{0}+e^{+} e^{-} h^{0}$ \\
\hline 920 & $\ell^{+} \ell^{-} \rightarrow \nu_{e} \bar{\nu}_{e} H^{0}+e^{+} e^{-} H^{0}$ \\
\hline 960 & $\ell^{+} \ell^{-} \rightarrow Z^{0} h^{0}$ \\
\hline 970 & $\ell^{+} \ell^{-} \rightarrow Z^{0} H^{0}$ \\
\hline 955 & $\ell^{+} \ell^{-} \rightarrow H^{+} H^{-}$ \\
\hline 965 & $\ell^{+} \ell^{-} \rightarrow A^{0} h^{0}$ \\
\hline 965 & $\ell^{+} \ell^{-} \rightarrow A^{0} H^{0}$ \\
\hline
\end{tabular}

Table 6: The MSSM hard scattering processes implemented in HERWIG (continued on following pages). 


\begin{tabular}{|c|l|}
\hline IPROC & Process \\
\hline $3000-999$ & R-parity conserving SUSY processes \\
3000 & 2-parton $\rightarrow$ 2-sparticle processes (sum of those below) \\
3010 & 2-parton $\rightarrow$ 2-sparton processes \\
3020 & 2-parton $\rightarrow$ 2-gaugino processes \\
3030 & 2-parton $\rightarrow 2$-slepton processes \\
\hline $3100+\mathrm{ISQ}$ & $g g / q \bar{q} \rightarrow \tilde{q} \tilde{q}^{*} H^{ \pm}$(cf. ref. [1] for definition of ISQ) \\
\hline $3200+\mathrm{ISQ}$ & $g g / q \bar{q} \rightarrow \tilde{q} \tilde{q}^{*} h, H, A$ (") \\
\hline 3310,3315 & $q \bar{q}^{\prime} \rightarrow W^{ \pm} h^{0}, H^{ \pm} h^{0}$ \\
3320,3325 & $q \bar{q}^{\prime} \rightarrow W^{ \pm} H^{0}, H^{ \pm} H^{0}$ \\
3335 & $q \bar{q}^{\prime} \rightarrow H^{ \pm} A^{0}$ \\
3350 & $q \bar{q} \rightarrow W^{ \pm} H^{\mp}$ \\
3355 & $q \bar{q} \rightarrow H^{ \pm} H^{\mp}$ \\
3360,3365 & $q \bar{q} \rightarrow Z^{0} h^{0}, A^{0} h^{0}$ \\
3370,3375 & $q \bar{q} \rightarrow Z^{0} H^{0}, A^{0} H^{0}$ \\
\hline 3410 & $b g \rightarrow b h^{0}+$ ch. conj. \\
3420 & $b g \rightarrow b H^{0}+$ ch. conj. \\
3430 & $b g \rightarrow b A^{0}+$ ch. conj. \\
3450 & $b g \rightarrow t H^{-}+$ch. conj. \\
\hline 3500 & $b q \rightarrow b q^{\prime} H^{ \pm}+$ch. conj. \\
\hline 3610 & $q \bar{q} / g g \rightarrow h^{0}$ \\
3620 & $q \bar{q} / g g \rightarrow H^{0}$ \\
3630 & $q \bar{q} / g g \rightarrow A^{0}$ \\
\hline 3710 & $q \bar{q} \rightarrow q^{\prime} \bar{q}^{\prime} h^{0}$ \\
3720 & $q \bar{q} \rightarrow q^{\prime} \bar{q}^{\prime} H^{0}$ \\
\hline $3810+\mathrm{IQ}$ & $g g+q \bar{q} \rightarrow Q \bar{Q} h^{0}$ (IQ for $Q$ flavour) \\
$3820+\mathrm{IQ}$ & $g g+q \bar{q} \rightarrow Q \bar{Q} H^{0}(")$ \\
$3830+\mathrm{IQ}$ & $g g+q \bar{q} \rightarrow Q \bar{Q} A^{0}$ (") \\
3839 & $g g+q \bar{q} \rightarrow b \bar{t} H^{+}+$ch. conjg. \\
$3840+\mathrm{IQ}$ & $g g \rightarrow Q \bar{Q} h^{0}(\mathrm{IQ}$ as above) \\
$3850+\mathrm{IQ}$ & $g g \rightarrow Q \bar{Q} H^{0}(")$ \\
$3860+\mathrm{IQ}$ & $g g \rightarrow Q \bar{Q} A^{0}(")$ \\
3869 & $g g \rightarrow b \bar{t} H^{+}+$ch. conjg. \\
$3870+\mathrm{IQ}$ & $q \bar{q} \rightarrow Q \bar{Q} h^{0}(\mathrm{IQ}$ as above) \\
$3880+\mathrm{IQ}$ & $q \bar{q} \rightarrow Q \bar{Q} H^{0}$ (") \\
\hline $3890+Q \bar{Q} A^{0} \quad$ ") \\
\hline
\end{tabular}

Table 6: continued. 


\begin{tabular}{|c|c|}
\hline IPROC & Process \\
\hline $4000-99$ & R-parity violating SUSY processes via LQD \\
\hline 4000 & single sparticle production, sum of $4010-4050$ \\
\hline 4010 & $\bar{u}_{j} d_{k} \rightarrow \widetilde{\chi}^{0} l_{i}^{-}, \bar{d}_{j} d_{k} \rightarrow \widetilde{\chi}^{0} \nu_{i}$ (all neutralinos) \\
\hline $4010+\mathrm{IN}$ & $\bar{u}_{j} d_{k} \rightarrow \widetilde{\chi}_{\mathrm{IN}}^{0} l_{i}^{-}, \bar{d}_{j} d_{k} \rightarrow \widetilde{\chi}_{\mathrm{IN}}^{0} \nu_{i}(\mathrm{IN}=$ neutralino mass state $)$ \\
\hline 4020 & $\bar{u}_{j} d_{k} \rightarrow \tilde{\chi}^{-} \nu_{i}, \bar{d}_{j} d_{k} \rightarrow \tilde{\chi}^{-} e_{i}^{+}$(all charginos) \\
\hline $4020+\mathrm{IC}$ & $\bar{u}_{j} d_{k} \rightarrow \tilde{\chi}_{\mathrm{IC}}^{-} \nu_{i}, \bar{d}_{j} d_{k} \rightarrow \tilde{\chi}_{\mathrm{IC}}^{-} e_{i}^{+} \quad(\mathrm{IC}=$ chargino mass state $)$ \\
\hline 4040 & $u_{j} \bar{d}_{k} \rightarrow \tilde{\tau}_{i}^{+} Z^{0}, u_{j} \bar{d}_{k} \rightarrow \widetilde{\nu}_{i} W^{+}$and $d_{j} \bar{d}_{k} \rightarrow \widetilde{\ell}_{i}^{+} W^{-}$ \\
\hline 4050 & $u_{j} \bar{d}_{k} \rightarrow \widetilde{\ell}_{i}^{+} h^{0} / H^{0} / A^{0}, u_{j} \bar{d}_{k} \rightarrow \widetilde{\nu}_{i} H^{+}$and $d_{j} \bar{d}_{k} \rightarrow \widetilde{\ell}_{i}^{+} H^{-}$ \\
\hline 4060 & Sum of 4070 and 4080 \\
\hline 4070 & $\bar{u}_{j} d_{k} \rightarrow \bar{u}_{l} d_{m}$ and $\bar{d}_{j} d_{k} \rightarrow \bar{d}_{l} d_{m}$, via LQD only \\
\hline 4080 & $\bar{u}_{j} d_{k} \rightarrow \nu_{j} l_{k}^{-}$and $\bar{d}_{j} d_{k} \rightarrow l_{j}^{+} l_{k}^{-}$, via LQD and LLE \\
\hline $4100-99$ & R-parity violating SUSY processes via UDD \\
\hline 4100 & single sparticle production, sum of $4110-4150$ \\
\hline 4110 & $u_{i} d_{j} \rightarrow \tilde{\chi}^{0} \bar{d}_{k}, d_{j} d_{k} \rightarrow \tilde{\chi}^{0} \bar{u}_{i}$ (all neutralinos) \\
\hline $4110+\mathrm{IN}$ & $u_{i} d_{j} \rightarrow \widetilde{\chi}_{\mathrm{IN}}^{0} \bar{d}_{k}, d_{j} d_{k} \rightarrow \widetilde{\chi}_{\mathrm{IN}}^{0} \bar{u}_{i}(\mathrm{IN}$ as above $)$ \\
\hline 4120 & $u_{i} d_{j} \rightarrow \tilde{\chi}^{+} \bar{u}_{k}, d_{j} d_{k} \rightarrow \tilde{\chi}^{-} \bar{d}_{i}$ (all charginos) \\
\hline $4120+\mathrm{IC}$ & $u_{i} d_{j} \rightarrow \tilde{\chi}_{\mathrm{IC}}^{+} \bar{u}_{k}, d_{j} d_{k} \rightarrow \tilde{\chi}_{\mathrm{IC}}^{-} \bar{d}_{i}(\mathrm{IC}$ as above $)$ \\
\hline 4130 & $u_{i} d_{j} \rightarrow \underset{\sim}{\widetilde{g}} \bar{d}_{k}, d_{j} d_{k} \rightarrow \widetilde{g} \bar{u}_{i}$ \\
\hline 4140 & $u_{i} d_{j} \rightarrow \tilde{b}_{\sim}^{*} Z^{0}, d_{j} d_{k} \rightarrow \tilde{t}_{1}^{*} Z^{0}, u_{i} d_{j} \rightarrow \tilde{t}_{i}^{*} W^{+}$and $d_{j} d_{k} \rightarrow \tilde{b}_{i}^{*} W^{-}$ \\
\hline 4150 & $\begin{array}{l}u_{i} d_{j} \rightarrow \tilde{d}_{k 1}^{*} h^{0} / H^{0} / A^{0}, d_{j} d_{k} \rightarrow \tilde{u}_{i 1}^{*} h^{0} / H^{0} / A^{0}, u_{i} d_{j} \rightarrow \tilde{u}_{k \alpha}^{*} H^{+}, \\
d_{j} d_{k} \rightarrow \tilde{d}_{i \alpha}^{*} H^{-}\end{array}$ \\
\hline 4160 & $u_{i} d_{j} \rightarrow u_{l} d_{m}, d_{j} d_{k} \rightarrow d_{l} d_{m}$ via $\mathrm{UDD}$ \\
\hline
\end{tabular}

Table 6: concluded.

\section{References}

[1] G. Corcella, I.G. Knowles, G. Marchesini, S. Moretti, K. Odagiri, P. Richardson, M.H. Seymour and B.R. Webber, HERWIG 6: an event generator for hadron emission reactions with interfering gluons (including supersymmetric processes), J. High Energy Phys. 01 (2001) 010 hep-ph/0011363]; HERWIG 6.1 release note, hep-ph/9912396; HERWIG 6.3 release note, hep-ph/0107071; HERWIG 6.4 release note, hep-ph/0201201

[2] H. Baer, V. Barger, D. Karatas and X. Tata, Detecting gluinos at hadron supercolliders, Phys. Rev. D 36 (1987) 96;

H. Baer, F.E. Paige, S.D. Protopopescu and X. Tata, ISAJET 7.48: a Monte Carlo event generator for $p p, \bar{p} p$ and $e^{+} e^{-}$reactions, hep-ph/0001086.

[3] A. Djouadi, J. Kalinowski and M. Spira, Hdecay: a program for Higgs boson decays in the standard model and its supersymmetric extension, Comput. Phys. Commun. 108 (1998) 56 hep-ph/9704448.

[4] H.E. Haber and G.L. Kane, The search for supersymmetry: probing physics beyond the standard model, Phys. Rept. 117 (1985) 75.

[5] J.F. Gunion and H.E. Haber, Higgs bosons in supersymmetric models, 1, Nucl. Phys. B 272 (1986) 1, erratum ibid. B402 (1993) 567. 
[6] J.F. Gunion, H.E. Haber, G.L. Kane and S. Dawson, The Higgs hunter's guide, Addison-Wesley, Reading USA 1990; Errata for the Higgs hunter's guide, hep-ph/9302272.

[7] S. Mrenna, SPYTHIA, a supersymmetric extension of PYTHIA 5.7, Comput. Phys. Commun. 101 (1997) 232 hep-ph/9609360;

T. Sjøstrand, L. Lonnblad and S. Mrenna, PYTHIA 6.2: physics and manual, hep-ph/0108264.

[8] S. Katsanevas and P. Morawitz, SUSYGEN 2.2: a Monte Carlo event generator for MSSM sparticle production at $e^{+} e^{-}$colliders, Comput. Phys. Commun. 112 (1998) 227 hep-ph/9711417.

[9] H. Baer, F.E. Paige, S.D. Protopopescu and X. Tata, ISAJET 7.48: a Monte Carlo event generator for $p p, \bar{p} p$ and $e^{+} e^{-}$reactions, hep-ph/0001086.

[10] H. Baer, J. Sender and X. Tata, The search for top squarks at the Fermilab Tevatron collider, Phys. Rev. D 50 (1994) 4517 hep-ph/9404342.

[11] X. Tata, What is supersymmetry and how do we find it?, hep-ph/9706307.

[12] A. Bartl, W. Majerotto and W. Porod, Squark and gluino decays for large $\tan \beta$, Z. Physik C 64 (1994) 499.

[13] K. Odagiri, Colour connection structure of (supersymmetric) QCD $(2 \rightarrow 2)$ processes, J. High Energy Phys. 10 (1998) 006 hep-ph/9806531.

[14] V.D. Barger, R.W. Robinett, W.Y. Keung and R.J.N. Phillips, Production of gauge fermions at colliders, Phys. Lett. B 131 (1983) 372.

[15] M. Spira, QCD effects in Higgs physics, Fortschr. Phys. 46 (1998) 203 hep-ph/9705337.

[16] R. Kleiss and W.J. Stirling, Spinor techniques for calculating $p \bar{p} \rightarrow W^{ \pm} / Z^{0}+$ jets, Nucl. Phys. B 262 (1985) 235.

[17] P. Richardson, Spin correlations in Monte Carlo simulations, J. High Energy Phys. 11 (2001) 029 hep-ph/0110108.

[18] A.A. Barrientos Bendezu and B.A. Kniehl, $W^{ \pm} H^{\mp}$ associated production at the large hadron collider, Phys. Rev. D 59 (1999) 015009 hep-ph/9807480.

[19] S. Moretti and K. Odagiri, Production of charged Higgs bosons of the minimal supersymmetric standard model in b-quark initiated processes at the large hadron collider, Phys. Rev. D 55 (1997) 5627 hep-ph/9611374.

[20] H. Dreiner, P. Richardson and M.H. Seymour, Parton-shower simulations of R-parity violating supersymmetric models, J. High Energy Phys. 04 (2000) 008 hep-ph/9912407.

[21] H. Dreiner and S. Lola, Prepared for physics with $e^{+} e^{-}$linear colliders, The European Working Groups 4 Feb. - 1 Sep. 1995, session 3, Hamburg, Germany, 30 Aug-1 Sep 1995.

[22] G.F. Giudice et al., Searches for new physics, hep-ph/9602207.

[23] M. Chemtob and G. Moreau, Broken R-parity contributions to flavor changing rates and CP asymmetries in fermion pair production at leptonic colliders, Phys. Rev. D 59 (1999) 116012 hep-ph/9806494.

[24] R. Alanakyan, Scalar neutrinos and Higgs bosons production with photons at lepton-antilepton colliders, Mod. Phys. Lett. A 13 (1998) 3073 hep-ph/9709501; R-parity violation and scalar leptons production at lepton antilepton colliders, hep-ph/9804247.

[25] J. Kalinowski, R. Rückl, H. Spiesberger and P.M. Zerwas, Supersymmetry with R-parity breaking: contact interactions and resonance formation in leptonic processes at LEP2, Phys. Lett. B 406 (1997) 314 hep-ph/9703436. 